\title{
FROM VANISHING THEOREMS TO ESTIMATING THEOREMS: THE BOCHNER TECHNIQUE REVISITED
}

\author{
PIERRE H. BÉRARD \\ Dedicated to Marcel Berger on his 60th birthday
}

\section{CONTENTS}

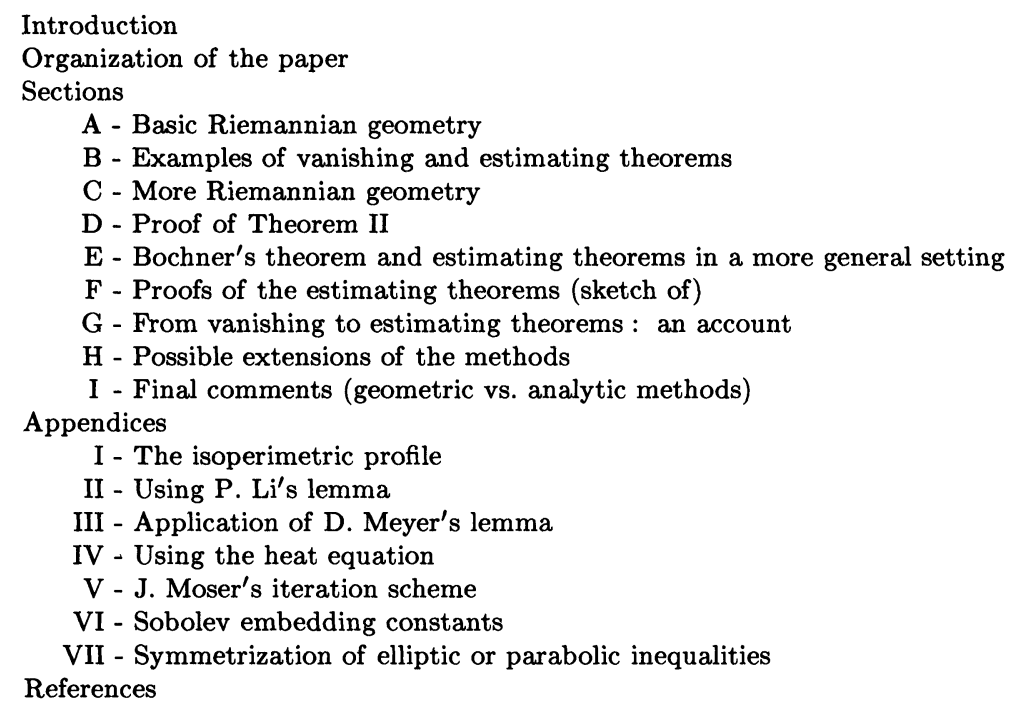

Introduction. In the 1940s, S. Bochner devised an analytic technique to obtain vanishing theorems for some topological or geometric invariants (e.g. Betti numbers, the dimension of the vector space of Killing vector-fields) on a closed (i.e. compact without boundary) Riemannian manifold, under some curvature assumption.

As a matter of fact, the word technique might be misleading. On the one hand it is not so easy to explain the technical details of the proofs in which S. Bochner's ideas are used and this is not our purpose here; on the other hand, the ideas are quite simple. Indeed, the basic idea is to show that some object (a harmonic form in the case of Betti numbers, a Killing vector-field,...) satisfies an elliptic inequality, provided that some curvature assumption is satisfied. The proofs then reduce to applying the maximum principle or to integrating over the manifold.

Received by the editors December 15, 1986 and, in revised form, February 4, 1988.

1980 Mathematics Subject Classification (1985 Revision). Primary 58G30, 53C20; Secondary 58G11, 58E99. 
The purpose of the present paper is two-fold:

(1) we want to describe some recent extensions of the Bochner technique; indeed, the original vanishing theorems have recently been generalized (in a rather general framework) to estimating theorems (see $\S \mathrm{B}$ for a typical statement and $\S \mathrm{E}$ for the main results);

(2) we also want to describe some technical tools involving analysis on a Riemannian manifold and isoperimetric inequalities: they might indeed be useful in other contexts.

In order to limit the necessary prerequisites we limit ourselves to the realm of Riemannian geometry (the reader not familiar with the basic concepts of Riemannian geometry is referred to $\S \S \mathrm{A}$ and $\mathrm{C}$ ). A more detailed presentation of the Bochner technique in differential geometry (in particular as applied to Kähler manifolds and to harmonic maps) is given in [Wu]; we refer to [Si1, 2 and Sh-So] for vanishing theorems in complex geometry.

The oldest vanishing (and estimating) theorem goes back to C. F. Gauss and O. Bonnet for compact oriented Riemannian surfaces without boundary. For such a surface,

$$
\int_{M} K(m) d a(m)=2 \pi \chi(M)
$$

where $K$ is the Gaussian curvature (the product of the principal curvatures; see also $\S \mathrm{A}), d a$ is the natural element of area on $M$ and $\chi(M)$ is the Euler characteristic (a topological invariant which is equal to two minus twice the number of holes).

VANISHING THEOREM. If $K \geq 0$ and $K \not \equiv 0$ then $\chi(M)>0$, i.e. $M$ is homeomorphic to $S^{2}$.

Estimating TheOREM. If $K \geq-k$ and $\operatorname{Area}(M) \leq V(k, V$ positive constants) then $|\chi(M)| \leq \max \{1, k V /(2 \pi)\}$ : there are only finitely many surfaces admitting a Riemannian metric satisfying the preceding inequalities.

By the Gauss-Bonnet theorem the Euler characteristic can be viewed as a topological obstruction to the existence of certain Riemannian metrics on surfaces (the sphere whose Euler characteristic is 2 does not carry a metric with nonpositive Gaussian curvature; the torus whose Euler characteristic is 0 does not carry a metric with positive Gaussian curvature).

The Gauss-Bonnet theorem has been generalized to higher dimensions, involving certain notions of curvature and characteristic classes [Ko-No].

The above estimating theorem has been generalized to higher dimensions by A. Weinstein (1967) and J. Cheeger (1970). Given a positive integer $n$ and positive constants, $V, D$, there are up to homeomorphism finitely many compact $n$-dimensional smooth manifolds without boundary admitting a Riemannian metric $g$ such that $\left|\sigma_{g}\right| \leq 1$ (the sectional curvature of $(M, g)$; see $\S \mathrm{A})$, Volume $(M, g) \geq V$ and $\operatorname{Diameter}(M, g) \leq D$. One can actually count the number of homeomorphism types (S. Peters 1984). In particular, any topological invariant on an $n$-dimensional Riemannian manifold satisfying the preceding inequalities is bounded; the topological types involved are not known and the finiteness theorem does not provide bounds on the topological invariants either. It is therefore natural to look for explicit bounds. 
Introducing a Hausdorff distance between Riemannian manifolds (compact without boundary; this distance generalizes the Hausdorff distance between compact subsets of a given metric space), M. Gromov (1980) proved that the set of $n$-dimensional Riemannian manifolds which satisfy the assumptions of the Cheeger-Weinstein finiteness theorem is compact. Under weaker assumptions (a lower bound on the Ricci curvature-a mean value of sectional curvatures; see $\S \mathrm{A}$ - and an upper bound on the diameter) M. Gromov also proved a precompactness theorem for the Hausdorff distance between Riemannian manifolds. It is again natural to try to bound the topological or geometric invariants under geometric assumptions (i.e. on curvature, diameter, volume, dimension...).

The estimating theorems which are described in $\S \mathrm{E}$ give a partial answer to this problem; in particular they give bounds on the Betti numbers depending on $n$ (dimension), 1 (an upper bound on $\left|\sigma_{g}\right|$ by scaling) and $D$ (an upper bound on the diameter).

For more details on the topics of finiteness theorems and compactness theorems we refer to $[\mathbf{P a}$ and $\mathbf{S a}]$.

Organization of the paper. Basic notions from Riemannian geometry are given in $\S \S \mathrm{A}$ and $\mathrm{C}$. We then start from two examples of vanishing theorems (both due to S. Bochner): Theorems I and II in $\S \mathrm{B}$; we explain their geometric meaning, relate them to natural problems and we state a first instance of an estimating theorem: $\S \mathrm{B}$, Theorem III. The proof of Theorem II is sketched in $\S \mathrm{D}$.

In $\S \mathrm{E}$ we describe a general setting and a general vanishing theorem of Bochner type (Theorem IV). Natural questions are asked which lead us to our general estimating theorems (Theorems V and VI). Proofs are sketched in $\S \mathrm{F}$. Behind the ideas of the proofs there are also some techniques which might be useful elsewhere. These techniques and the detailed proofs of Theorems V and VI $(\S \mathrm{E})$ are described in several appendices which are quite independent from the main text.

$\S \S \mathrm{G}$ and $\mathrm{H}$ give respectively a historical sketch and possible extensions of the methods we describe in this paper.

In $\S I$ we sketch a comparison between analytic techniques and geometric techniques in the part of Riemannian geometry dealing with curvature and topology.

In an attempt to make this text usable by nonspecialists, including graduate students, we have provided $\S \S \mathrm{A}$ and $\mathrm{C}$ (topics from Riemannian geometry) and we have tried to give complete proofs (Appendices) or references.

ACKNOWLEDGEMENT. This paper grew out of lectures given in Brazil in August 1985. I would like to thank the colleagues, both in Brazil and in France, who helped me turn a rough set of notes into a paper; special thanks are due to G. Besson and J. P. Bourguignon.

I gratefully acknowledge the financial support of Brazil's $\mathrm{CNP}_{q}$ (at IMPA) and France's CNRS during the preparation of this paper.

A. Basic Riemannian geometry. In this section we recall some basic concepts in Riemannian geometry. We need them to state the vanishing and 
estimating theorems we will deal with in this paper. Further concepts will be introduced when we sketch the proofs $(\S \mathrm{C})$. Possible references are [B-G-M, Che-Eb, Sa].

Let $(M, g)$ be a Riemannian manifold, i.e. a manifold ${ }^{1} M$ with a Riemannian metric $g$ : a collection of inner products; $g_{x}$ is an inner product in the tangent space $T_{x} M$, depending smoothly on the point $x$.

A nice example of a Riemannian manifold is that of a submanifold $M$ in Euclidean space with Riemannian metric $g_{x}$ in $T_{x} M$ induced by the Euclidean inner product.

The Riemannian distance between two points $x$ and $y$ in $M$ is defined to be the infimum of the length (w.r.t. $g$ ) of the curves from $x$ to $y$. The diameter $D(g)$ of $(M, g)$ is the diameter of $M$ for the Riemannian distance.

The geodesics are the curves which satisfy the Euler-Lagrange equation of this minimization problem: they satisfy a second order nonlinear differential equation on $M$. In particular, given any point $x$ in $M$ and any unit vector $u$ in $T_{x} M$, there is one and only one geodesic $c_{x, u}$ parametrized w.r.t. arc-length such that $c_{x, u}(0)=x$ and $\dot{c}_{x, u}(0)=u$ (such a geodesic is defined for all values of $t$ when $M$ is closed). We define the exponential map $\exp _{x}$ from $T_{x} M$ to $M$ by $\exp _{x}(t u)=c_{x, u}(t)$, for any nonnegative $t$ and any unit tangent vector $u$ in $T_{x} M$. The exponential map is a local diffeomorphism from a neighborhood of 0 in $T_{x} M$ onto a neighborhood of $x$ in $M$ (w.r.t. the Riemannian distance): indeed, its derivative at 0 is the identity map.

An isometry between two Riemannian manifolds $(M, g)$ and $(N, h)$ is a map which induces linear isometries between the tangent spaces w.r.t. the inner products $g$ and $h$ respectively. The various notions of curvature measure how the exponential maps differ from being isometries (at least locally). Let $P$ be a 2-plane in $T_{x} M$. Given a small enough $r$, consider the image under $\exp _{x}$ of the circle of radius $r$ and with center 0 in the plane $P$. This is a closed curve in $M$ whose length we denote by $L(r)$. When $r$ goes to zero, we have Puiseux' formula

$$
L(r)=2 \pi r\left(1-\frac{1}{6} \sigma(x, P) r^{2}+O\left(r^{3}\right)\right) .
$$

The number $\sigma(x, P)$ is called the sectional curvature of the 2-plane $P$ at $x$. If $(M, g)$ is an immersed surface in $\mathbf{R}^{3}$, with induced metric in each tangent space, the sectional curvature of the tangent plane coincides with the Gaussian curvature of the surface (i.e. the product of the principal curvatures).

It is clear that the sectional curvatures of the Euclidean space $\mathbf{R}^{n}$ are constant equal to 0 . The sectional curvatures of the sphere $S^{n}$ of radius one in $\mathbf{R}^{n+1}$, with induced metric, are constant equal to +1 (indeed the curve considered above is always an ordinary circle with radius $\sin r$ ). Here is an example of a Riemannian manifold with sectional curvatures -1 . Let $\left(H^{n}, g_{H}\right)$ be the ball of radius 2 in $\mathbf{R}^{n}$, with the metric $g_{H}(U, V)=\left(1-|x|^{2} / 4\right)^{-2}(U, V)$ at the point $x$, where $(U, V)$ is the Euclidean inner product of the tangent vectors $U$ and $V$ and $|x|$ is the Euclidean norm of $x$. It is not difficult to check

\footnotetext{
${ }^{1}$ Throughout this text we will use the word "manifold" as a short cut for "closed, connected, smooth $n$-dimensional manifold", except in very few instances in which the context will be clear. Closed means compact without boundary.
} 
that the geodesics issued from 0 are carried by the Euclidean lines through 0 and that the sectional curvatures at 0 are constant equal to -1 . As a matter of fact, $\left(H^{n}, g_{H}\right)$ has constant sectional curvatures equal to -1 because the homographies form a transitive group of isometries.

A Riemannian manifold is also equipped with a natural Riemannian measure $v_{g}$ whose expression in a local coordinate system $\left\{x_{i}\right\}$ is $\operatorname{det}\left(g_{i j}\right)^{1 / 2} d x$, where $d x$ is the Lebesgue measure and where $g_{i j}=g\left(\partial / \partial x_{i}, \partial / \partial x_{j}\right)$. The volume of $(M, g)$ is denoted by $V(g)\left(=\int_{M} d v_{g}\right)$.

In order to be able to state our vanishing theorems, we need another notion of curvature which we now define. We can write the pull-back $\exp _{x}^{*} v_{g}$ of the Riemannian measure $v_{g}$ by $\exp _{x}$ in polar coordinates in $T_{x} M: \exp _{x}^{*} v_{g}=$ $\theta_{x}(t, u) d t d u$, where $t \geq 0, u$ is a unit vector and $d u$ is the canonical measure on the unit sphere. When $t$ goes to zero, we have the formula

$$
\theta_{x}(t, u)=t^{n-1}\left(1-\frac{1}{6} \rho_{x}(u) t^{2}+O\left(t^{3}\right)\right) .
$$

The number $\rho_{x}(u)$ is a quadratic form on $T_{x} M$ which defines a symmetric bilinear form called the Ricci curvature, $\operatorname{Ric}_{g}$, of $M$ at the point $x$. If $\left\{u, e_{2}, \ldots, e_{n}\right\}$ is an orthonormal basis in $T_{x} M$ and if $P_{i}$ is the 2-plane spanned by $u$ and $e_{i}$, we also have the formula

$$
\operatorname{Ric}_{g}(u, u)=\sum_{i=2}^{n} \sigma\left(x, P_{i}\right) .
$$

Further concepts from Riemannian geometry will be introduced in $\S \mathrm{C}$.

Let us for the moment proceed with examples of vanishing and estimating theorems.

\section{B. Examples of vanishing and estimating theorems.}

THEOREM I [Bo, Bo-Ya]. Let $(M, g)$ be a Riemannian manifold.

(i) If the Ricci curvature of $M$ is nonpositive, then the dimension of the vector space of Killing vector-fields is less than or equal to the dimension of $M$.

(ii) If the Ricci curvature of $M$ is nonpositive and negative definite at some point, then there are no nontrivial Killing vector-fields.

Recall that a Killing vector-field on a Riemannian manifold is a vector-field which generates a one-parameter group of isometries.

THEOREM II [Bo, Bo-Ya]. Let $(M, g)$ be a Riemannian manifold.

(i) If the Ricci curvature of $M$ is nonnegative then the first Betti number of $M$ is less than or equal to the dimension of $M$.

(ii) If the Ricci curvature of $M$ is nonnegative and positive definite at some point then the first Betti number is equal to zero.

Recall that the first Betti number is equal to the dimension of the quotient vector space of closed differential 1 -forms modulo exact 1 -forms; this is a topological invariant (which is twice the genus in dimension two, i.e. twice the number of holes).

CONSEQUENCES. (I) Under the assumption of Theorem I(i) we see that the (compact) Lie group $\operatorname{Isom}(M, g)$ of isometries of $(M, g)$ has dimension $r$ not 
greater than the dimension of $M$ (one can also prove that the universal cover of $M$ splits orthogonally as $M^{\prime} \times \mathbf{R}^{r}$ where $M^{\prime}$ is simply-connected). Under the assumption of Theorem I(ii) we see that the isometry group of $(M, g)$ is finite. This means that nonpositive Ricci curvature is an obstruction to the existence of "many" isometries (i.e. internal symmetries of the metric).

(II) From Theorem II, we deduce that the first Betti number is a topological obstruction to the existence of metrics with positive or nonnegative Ricci curvature. For example, the $n$-torus (whose first Betti number is equal to $n$ ) does not carry a Riemannian metric with positive Ricci curvature. One can also view Theorem II as saying that a closed manifold which admits a metric of nonnegative Ricci curvature has a simple topological type in some sense (namely, its first Betti number is not greater than the dimension).

These observations are simple instances of partial answers to the following general problems (compare with the motivations given in the Introduction).

PROBLEM (a). Find obstructions (e.g. topological) for the existence of special metrics on a given manifold $M$ (e.g. metrics one of whose curvatures satisfies a sign condition).

PROBLEM (b). Find restrictions on the global behaviour (e.g. bound some topological invariant) of a manifold which carries a metric satisfying some condition (e.g. a metric whose sectional curvatures are bounded, together with some scaling assumption).

Much work has been devoted to these problems in recent years. Some of the methods which have been used involve ideas related to the original Bochner technique as illustrated by Theorems I and II above; other methods involve more geometric tools together with analysis: we refer to the chart in $\S \mathrm{I}$. In the spirit of Theorem II, one can prove the following estimating theorem which gives a partial answer to Problem (b).

THEOREM. III. Given an n-dimensional Riemannian manifold $(M, g)$, let $r_{\min }(g)$ denote the infimum of the Ricci curvature $\mathrm{Ric}_{g}$ on the unit tangent bundle.

(i) There exists a positive number $\varepsilon(n)$ such that $r_{\min }(g) D^{2}(g) \geq-\varepsilon(n)$ implies $b_{1}(M) \leq n\left(b_{1}(M)\right.$ is the 1 st Betti number, $D(g)$ the diameter of $M, g)$ ).

(ii) For any $k \in \mathbf{R}_{+}$, there exists a number $A(n, k)$ such that $r_{\min }(g) D^{2}(g) \geq$ $-k^{2}$ implies that $b_{1}(M) \leq A(n, k)$.

Comments. (a) Part (i) of Theorem III is a kind of semicontinuity property of the estimate in Theorem II(i).

(b) Given a Riemannian manifold $(M, g)$, one can always make dilations by multiplying the metric by some positive number; these dilations change the curvature but obviously do not change the topology of $M$. In Theorem III, we use the diameter $D(g)$ to scale the metric (the quantity $r_{\min }(g) D^{2}(g)$ is invariant under dilations). It is not possible in general to scale the metric with the volume $V(g)$ of $(M, g)$; see [Bé-Ga] (except in dimension 2 as the Gauss-Bonnet theorem shows). 
Theorem III is a special instance of the vanishing theorems which will be stated in $\S \mathrm{E}$. Before we proceed with the proof of Theorem II $(\S \mathrm{D})$, we need more concepts from Riemannian geometry.

C. More Riemannian geometry. Given a Riemannian manifold $(M, g)$ an important concept is that of the natural covariant derivative (or LeviCivita connection): to the vector-fields $X, Y$ we associate another vector-field $D_{X} Y$, the covariant derivative of $Y$ w.r.t. $X$. We will not give the formal definition (see for example [B-G-M]) but let us mention the following fact. Let $(M, g)$ be an immersed submanifold of $\mathbf{R}^{N}$, with induced metric. Let $X$ and $Y$ be tangent vector-fields on $M$. We can write $Y=\left(y_{1}, \ldots, y_{N}\right)$ in the canonical basis of $\mathbf{R}^{N}$ and differentiate each component $y_{i}$ in the direction of $X$. This gives a new vector-field $Y_{X}$ along $M: Y_{X}=\left(X \cdot y_{1}, \ldots, X \cdot y_{N}\right)$. The covariant derivative $D_{X} Y$ of $Y$ w.r.t. $X$ is the part of $Y_{X}$ tangential to $M$, i.e. the orthogonal projection (in $\mathbf{R}^{N}$ ) of $Y_{X}$ onto the tangent space of $M$ at each point.

One can extend $D$ to a covariant derivative of tensor-fields w.r.t. vectorfields. For differential 1-forms this is done as follows. The covariant derivative $D \alpha$ of the 1-form $\alpha$ is defined by $\left(D_{X} \alpha\right)(Y)=X \cdot(\alpha(Y))-\alpha\left(D_{X} Y\right)$ for any vector-fields $X$ and $Y$ (i.e. the extension is such that the Leibnitz rule for the derivative of a product is true).

The Riemannian metric $g$ defines a natural identification between $T M$ and $T^{*} M$. We use this pairing to put an inner product on tensors, for example on $\bigwedge^{p} T_{x}^{*} M$. This gives us a natural $L^{2}$-structure on smooth tensor fields, differential $p$-forms, ... (using the Riemannian measure $v_{g}$ ). We define the codifferential $\delta$ to be the formal adjoint of the exterior derivative $d$ on $C^{\infty}\left(\bigwedge^{*} T^{*} M\right)$ w.r.t. these $L^{2}$-structures. The Hodge-de Rham Laplacian on $p$-forms is defined to be $\Delta_{H}=d \circ \delta+\delta \circ d$. The Riemannian Laplacian $\Delta$ is the Hodge-de Rham Laplacian on 0 -forms (i.e. on functions). Similarly, one can define the formal adjoint $D^{*}$ of $D$, say on 1-forms $D: C^{\infty}\left(T^{*} M\right) \rightarrow C^{\infty}\left(T^{*} M \otimes T^{*} M\right)$, and the rough (or Bochner) Laplacian $\bar{\Delta}=D^{*} D$. The operators $\Delta_{H}$ and $\bar{\Delta}$ are nonnegative elliptic second order linear differential operators. They turn out to have the same principal symbol and they only differ by zeroth order term. In the special case of differential 1 -forms, one has

$$
\Delta_{H} \alpha=\bar{\Delta} \alpha+\operatorname{Ric}\left(\alpha^{\#}, \bullet\right)
$$

where $\alpha^{\#}$ is the vector-field dual to the 1-form $\alpha$. Such a formula is now called a Weitzenböck formula. This formula comes from the fact that covariant derivatives of order at least three do not commute in general on a Riemannian manifold: indeed, the curvature is precisely the obstruction to commuting derivatives [Bé, II.52]. For a proof of $(*)$ see [Bo-Ya, Wu or Bé, §VI.12].

D. Proof of Theorem II. By the Hodge-de Rham theorem [Ee], one can parametrize the equivalence classes of closed 1-forms modulo exact 1 -forms by harmonic 1-forms $\left(\Delta_{H} \alpha=0\right)$ : the first Betti number is equal to the dimension of the kernel of the Hodge-de Rham Laplacian $\Delta_{H}$ acting on 1-forms. Let $\alpha$ be a harmonic 1-form. The Weitzenböck formula $(*)$ in the preceding section implies that $(\bar{\Delta} \alpha, \alpha)=-\operatorname{Ric}\left(\alpha^{\#}, \alpha^{\#}\right)$. 
Integrating over the manifold $M$ and using the definition of $\bar{\Delta}$, we conclude that

$$
\int_{M}|D \alpha|^{2} v_{g}=-\int_{M} \operatorname{Ric}\left(\alpha^{\#}, \alpha^{\#}\right) v_{g}
$$

The assumption Ric $\geq 0$ implies that $D \alpha \equiv 0$ : we say that the 1 -form $\alpha$ is parallel. Given any curve $c(t)$ in $M$, one can construct a coframe $\omega_{1}, \ldots, \omega_{n}$ along $c(t)$ such that $D_{\dot{c}(t)} \omega_{i}=0,1 \leq i \leq n$ (first order differential equations along the curve). In such a parallel co-frame the components of the parallel form $\alpha$ are constant: this implies that we have at most $n$ parameters for $\alpha$, i.e. there are at most $n$ linearly independent parallel 1-forms. We therefore conclude that the 1st Betti number $b_{1}(M)$ satisfies $b_{1}(M) \leq n$ when Ric $\geq 0$. If furthermore $\operatorname{Ric}\left(x_{0}\right)>0$ for some $x_{0} \in M$ we have $\alpha\left(x_{0}\right)=0$ which implies (because $\alpha$ is parallel) that $\alpha \equiv 0$ and hence $b_{1}(M)=0$.

Another proof of Theorem II uses the equality $(\bar{\Delta} \alpha, \alpha)=-\operatorname{Ric}\left(\alpha^{\#}, \alpha^{\#}\right)$, the second Kato inequality $(\S \mathrm{E},(\mathrm{K} 2))$ and the maximum principle (see $[\mathbf{W u}])$.

E. Bochner's theorem in a more general setting. In this section, we introduce the general setting in which we will state the estimating theorems.

Recall that $(M, g)$ is an $n$-dimensional smooth closed connected Riemannian manifold with Riemannian measure $v_{g}$.

We now consider a Riemannian bundle $E$ over $M$, i.e. a fiber bundle $E$ equipped with a scalar product $\langle\cdot, \cdot\rangle_{x}$ in each fiber, depending $C^{\infty}$ on $x$. We assume furthermore that $E$ is equipped with a compatible connection $D$ (i.e. $X \cdot\langle s, t\rangle=\left\langle D_{X} s, t\right\rangle+\left\langle s, D_{X} t\right\rangle$, for all vector-field $X$ on $M$ and $C^{\infty}$ sections $s, t$ of $E$ ). The scalar products and the connections (of $E$, as well as of $M$ ) naturally extend to tensor products, and the extensions will be denoted by the same symbols: compare with $\S \mathrm{C}$ above or see [B-G-M].

We define the $L^{2}$-norm of a $C^{\infty}$ section $s$ of $E$ by

$$
\|s\|_{2}^{2}=\int_{M}\langle s, s\rangle_{x} v_{g}=\int_{M}|s|_{x}^{2} v_{g},
$$

and we introduce the associated Hilbert space $L^{2}(E)$. One can define similarly the spaces $L^{p}(E)$. For example, for a $C^{\infty}$ function $f$, one has $\|d f\|_{2}^{2}=$ $\int_{M}|d f|_{x}^{2} v_{g}$, where $|\cdot|_{x}$ is the norm in $T_{x}^{*} M$ given by the dual Riemannian metric $g^{*}$ on $T^{*} M ;\|d f\|_{2}$ is also the $L^{2}$-norm of the gradient of $f$.

We assume that $E$ is equipped with a "natural" Laplacian $\widetilde{\Delta}: \widetilde{\Delta}$ is a 2 nd order elliptic linear differential operator on $C^{\infty}(E)$ which is symmetric with respect to the $L^{2}(E)$-inner product: $\langle\langle\widetilde{\Delta} s, t\rangle\rangle=\int_{M}\langle\widetilde{\Delta} s, t\rangle_{x} v_{g}=\langle\langle s, \widetilde{\Delta} t\rangle\rangle$, for all $C^{\infty}$-sections $s$ and $t$.

Using the $L^{2}$-structures, one can also define the rough (or Bochner) Laplacian on $C^{\infty}(E)$ by $\bar{\Delta}=D^{*} D$, where $D^{*}$ is the formal-adjoint of the differential operator $D: C^{\infty}(E) \rightarrow C^{\infty}\left(T^{*} M \otimes E\right)$.

Our final (and main) assumption is that $\widetilde{\Delta}$ satisfies the following Weitzenböck formula:

$$
\widetilde{\Delta} s=\bar{\Delta} s+\mathscr{R} s, \quad \text { for all } s \in C^{\infty}(E),
$$


where $\mathscr{R}$ is a symmetric endomorphism of $E$ : for all $x$ in $M, \mathscr{R}_{x}: E_{x} \rightarrow$ $E_{x}$ is a symmetric endomorphism (this symmetry property follows from the symmetry of $\widetilde{\Delta}$ and $\bar{\Delta}$ ).

Weitzenböck formulas play a very important role in geometry. We refer to [Bou, Ee-Le, La and Wu] for more details on this subject.

EXAMPLES. (i) $E=T^{*} M ; \widetilde{\Delta}=\Delta_{H}$, the Hodge-de Rham Laplacian on 1-forms;

(ii) $E=\bigwedge^{p} T^{*} M ; \widetilde{\Delta}=\Delta_{H}$, the Hodge-de Rham Laplacian on $p$-forms, $\mathscr{R}$ is expressed in terms of the curvature operator: see [Wu or Ga-Me];

(iii) $E=M \times \mathbf{R} ; \widetilde{\Delta}=\Delta-\lambda$, where $\Delta$ is the usual Laplacian on functions $\left(\Delta=-d^{2} / d x^{2}\right.$ on $\left.\mathbf{R}\right)$.

For other examples and comments see $\S \S \mathrm{H}$ and I.

Since $\widetilde{\Delta}$ is elliptic and $M$ is compact, the dimension $\delta(E)$ of the kernel of $\widetilde{\Delta}, \delta(E)=\operatorname{dim} \operatorname{Ker} \widetilde{\Delta}$, is finite. The proof of the following vanishing theorem is a verbatim reproduction of that of Theorem II.

THEOREM IV. Let $E$ be a Riemannian vector bundle of rank $l$ over the closed Riemannian manifold $(M, g)$. If the symmetric endomorphism $\mathscr{R}$ (formula (W)) satisfies

(i) $\mathscr{R} \geq 0$, then $\delta(E) \leq l$;

(ii) $\mathscr{R} \geq 0$, and there exists an $x_{0}$ in $M$ such that $\mathscr{R}\left(x_{0}\right)>0$, then $\delta(E)=0$.

We now ask the following questions:

QUESTIONS. (Q1) Can one slightly release the assumption $\mathscr{R} \geq 0$ in Theorem IV, and still obtain (possibly with some scaling assumption) $\bar{\delta}(E) \leq$ $l$ ?

(Q2) Can one give general upper bounds on $\delta(E)$ in terms of $\mathscr{R}$ (without the positivity assumption), and the geometry of $(M, g)$ ?

REMARK. The fact that some scaling is needed has already been explained in $\S \mathrm{B}$ (Comments following Theorem III).

Notations. For a closed Riemannian manifold $(M, g)$, we let

$D(g)=$ Diameter of $(M, g)$;

$V(g)=$ Volume of $(M, g)$;

Ric $_{g}=$ Ricci curvature of $(M, g)$;

$r_{g}(x)=\inf \left\{\operatorname{Ric}_{g}(u, u): u \in T_{x} M,|u|_{x}=1\right\}$;

$r_{\min }(g)=\inf \left\{r_{g}(x): x \in M\right\}$.

Similarly, for the endomorphism $\mathscr{R}$, we let

$\mathscr{R}(x)=\inf \left\{\langle\mathscr{R} s, s\rangle_{x}: s \in E_{x},|s|_{x}=1\right\}$;

$\mathscr{R}_{\text {min }}=\inf \{\mathscr{R}(x): x \in M\}$.

The following estimating theorems extend the original results of $\mathrm{S}$. Bochner ( $\S \mathrm{B}$, Theorems I and II) and give partial answers to the above questions (compare with §B, Theorem III).

THEOREM V. Let $\left(M^{n}, g\right)$ be a closed Riemannian manifold such that $r_{\min }(g) D^{2}(g) \geq(n-1) \varepsilon \alpha^{2}$, where $\alpha \in \mathbf{R}_{+}$and where $\varepsilon \in\{-1,0,1\}$. Let $E$ be a Riemannian vector bundle over $M$ as described above. Then there exists 
a positive number $A(n, \varepsilon, \alpha)$ such that

$$
\left(R_{\min } D^{2}(g)\right)_{-}<A(n, \varepsilon, \alpha) \Rightarrow \delta(E) \leq l
$$

( $x_{-}$is the negative part of $x \in \mathbf{R}: x_{-}=\sup (0,-x)$ ).

ThEOREM VI. Let $E \rightarrow\left(M^{n}, g\right)$ be as in Theorem V. Then, there exists a function $\Phi_{n, \varepsilon, \alpha}: \mathbf{R}_{+} \rightarrow \mathbf{R}_{+}$, such that

$$
\delta(E) \leq \Phi_{n, \varepsilon, \alpha}\left(\left(\mathscr{R}_{\min } D^{2}(g)\right)_{-}\right) .
$$

It should be pointed out that both the constant $A(n, \varepsilon, \alpha)$ and the function $\Phi_{n, \varepsilon, \alpha}$ are quite explicit and easily evaluated numerically.

Let us now give an idea on how to prove Theorems V and VI. As in the proof of S. Bochner's Theorem II, we use the Weitzenböck formula (W) to concentrate our attention on the rough Laplacian $\bar{\Delta}$. The following lemma enables us to reduce problems on sections of $E$ to problems on functions on M.

LEMMA (FIRST KATO INEQUALITY). Let $s$ be a smooth section of $E$. The (distributional) differential $d|s|$ of the pointwise norm $|s|$ of $s$ is in $L^{2}\left(T^{*} M\right)$ and satisfies the (distributional) inequality

$$
|d| s|| \leq|D s| \text {. }
$$

Proof. The first assertion is classical (see e.g. [Bé, VI.31]). For the second one, we write $(X$ any vector-field on $M) 2|s|(X \cdot|s|)=X \cdot|s|^{2}=2\left\langle D_{X} s, s\right\rangle$, and we apply the Cauchy-Schwarz inequality.

REMARKS. (i) The lemma goes back at least to T. Kato [Re-Si, Volume II, Chapter X]);

(ii) in the lemma, $|d| s||$ is the norm of the 1-form $d|s| ;|D s|$ is the norm of the section $D s$ of $T^{*} M \otimes E$.

For any smooth section $s$ of $E$, we can write $\Delta\left(|s|^{2}\right)=2|s| \Delta(|s|)-2|d| s||^{2}$, and, on the other hand, $\Delta\left(|s|^{2}\right)=2\langle\bar{\Delta} s, s\rangle-2|D s|^{2}$ (indeed the Laplacian can be written as $-\sum_{i=1}^{n}\left(D_{e_{i}} \circ D_{e_{i}}-D_{D_{e_{i}} e_{i}}\right)$ in any local orthonormal frame $\left.\left\{e_{i}\right\}_{i=1}^{n}\right)$.

From these equalities and the first Kato inequality we deduce the following

LEMMA (SECOND KATO INEQUALITY). With the preceding notations we have

$$
\left\{\begin{array}{l}
\text { For any } s \in C^{\infty}(E),|s| \Delta(|s|) \leq\langle\bar{\Delta} s, s\rangle, \\
\text { in the sense of distributions. }
\end{array}\right.
$$

F. Proofs of the estimating theorems (sketch of). In this section we sketch three methods for proving Theorems V and VI; full details are given in the Appendices. We use the notation of the preceding section and denote by $\mathscr{H}(\widetilde{\Delta})$ the vector-space of harmonic sections of $E(\widetilde{\Delta} s=0)$.

FIRST METHOD. A simple geometric lemma (see [Li, p. 460 or Appendix II]) shows that

$$
\text { (L) } \delta(E)=\operatorname{dim} \mathscr{H}(\widetilde{\Delta}) \leq l \sup \left\{\|s\|_{\infty}^{2} V(g) /\|s\|_{2}^{2}: s \in \mathscr{H}(\widetilde{\Delta}), s \neq 0\right\} .
$$

Recall that $l=\operatorname{rank}(E)$. 
By standard elliptic theory (de Giorgi-Nash-Moser iteration scheme) and using the second Kato inequality $\Delta(|s|) \leq\left(-\mathscr{R}_{\min }\right)|s|$, we deduce that the ratio $\|s\|_{\infty}^{2} V(g) /\|s\|_{2}^{2}$ (for any $s \in \mathscr{H}(\widetilde{\Delta})$ ) is bounded from above in terms of $\mathscr{R}_{\text {min }}$ and the Sobolev constant of the embedding $W^{1,2}(M, g) \rightarrow L^{2 n /(n-2)}$ $\left(M, v_{g}\right)$ (in the case in which $n \geq 3$ ), where $W^{1,2}(M, g)$ is the set of $L^{2}$ functions whose first derivatives are in $L^{2}$. The geometric assumption $r_{\min }(g)$ $D^{2}(g) \geq(n-1) \varepsilon \alpha^{2}$ allows us to estimate the Sobolev constant. We finally conclude that the ratio $\|s\|_{\infty}^{2} V(g) /\|s\|_{2}^{2}$ is bounded from above by a function $\Phi_{1}\left(n, \varepsilon, \alpha ; \mathscr{R}_{\min } D^{2}(g)\right)$.

Another method for estimating the ratio $\|s\|_{\infty}^{2} V(g) /\|s\|_{2}^{2}$ is to symmetrize the elliptic inequality $\Delta(|s|) \leq\left(-\mathscr{R}_{\min }\right)|s|$ on $M$ and to compare it with the elliptic equation $\Delta^{*} f=-\mathscr{R}_{\min } f$ on a model space $M^{*}$. This model space is constructed from geometric assumptions on $M$; in the case of the geometric assumption $r_{\min }(g) D^{2}(g) \geq(n-1) \varepsilon \alpha^{2}$, the model space $M^{*}$ is a sphere.

Both the estimate of the Sobolev constant and the symmetrization procedure depend on isoperimetric properties of $M$ (estimates on the isoperimetric constants or on the isoperimetric profile).

The implementation of our first method for proving Theorems V and VI (and related results) is fully described in the Appendices:

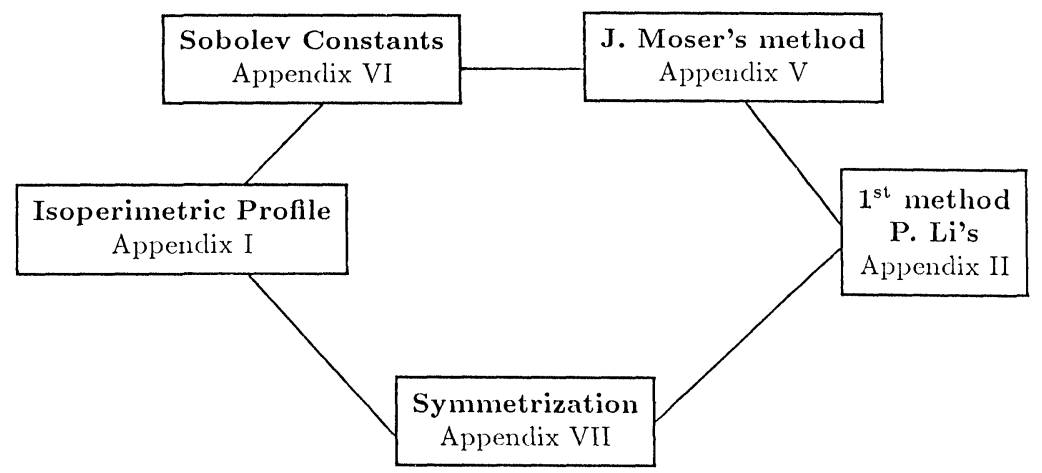

SECOND METHOD. By the Weitzenböck formula (W) and the first Kato inequality $(\mathrm{K} 1)$, any harmonic section $s \in \mathscr{H}(\widetilde{\Delta})$ satisfies the inequality

$$
\int_{M}|d| s||^{2} v_{g} \leq \int_{M}|D s|^{2} v_{g} \leq\left(-\mathscr{R}_{\min }\right) \int_{M}|s|^{2} v_{g}
$$

This inequality does not seem to say much because the first (i.e. least) eigenvalue of the Laplacian $\Delta$ acting on $C^{\infty}(M)$ is zero. In $\S \mathrm{D}$, we saw that any parallel section $s(D s=0)$ has constant norm and that parallel sections form a vector-space of dimension at most $l(=\operatorname{rank}(E))$. D. Meyer's lemma ([Me2] and Appendix III) gives a partial converse to this property. Roughly speaking the lemma says that given a vector-space of sections of $E$, of dimension at least $l+1$, there is at least one section whose norm is 
"far from being constant". In the case at hand we have the following: if $\operatorname{dim} \mathscr{H}(\widetilde{\Delta})=\delta(E) \geq l+1$ then there is a function $f_{0}$ on $M$ (related to the norm of a section) such that

$$
\int_{M}\left|d f_{0}\right|^{2} v_{g} \leq\left(-\mathscr{R}_{\min }\right) C_{l, n} \int_{M} f_{0}^{2} v_{g} \quad \text { and } \quad \int_{M} f_{0} v_{g}=0
$$

where $C_{l, n}$ is a universal positive constant. From this inequality we conclude that $\left(-\mathscr{R}_{\min }\right) C_{l, n} \geq \lambda_{2}(M, g)$, the second eigenvalue of $\Delta$ on $C^{\infty}(M)$. Theorem V now follows from a lower bound à la Cheeger for $\lambda_{2}(M, g)$; such a lower bound can be expressed in terms of $n, \varepsilon, \alpha$ and $D^{2}(g)$ from the geometric assumption $r_{\min }(g) D^{2}(g) \geq(n-1) \varepsilon \alpha^{2}$.

Our second method is described in Appendix III. It also depends on Appendices I and VI:

\begin{tabular}{|c|c|c|}
\hline $\begin{array}{c}\text { Isoperimetric Profile } \\
\text { Appendix I }\end{array}$ & $\begin{array}{c}\text { Sobolev Constants and } \lambda_{2} \\
\text { Appendix VI }\end{array}$
\end{tabular} \begin{tabular}{c}
$\begin{array}{c}\text { Second Method } \\
\text { (D. Meyer's) } \\
\text { Appendix III }\end{array}$ \\
\hline
\end{tabular}

THIRD METHOD. The second Kato inequality (K2) and the maximum principle for parabolic equations imply that one can bound from above the partition function $\operatorname{Tr}(\exp (-t \tilde{\Delta}))$ by $l \exp \left(-t \mathscr{R}_{\min }\right) \operatorname{Tr}(\exp (-t \Delta))$ (see [H-S$\mathrm{U}], l=\operatorname{rank}(E))$.

On the other hand, the geometric assumption $r_{\min }(g) D^{2}(g) \geq(n-1) \varepsilon \alpha^{2}$ implies that one can bound $\operatorname{Tr}(\exp (-t \Delta))$ from above by the partition function $\operatorname{Tr}\left(\exp \left(-t \Delta^{*}\right)\right)$ on a model space $M^{*}$ (in the case at hand a sphere): this is achieved by symmetrization.

Our third method is described in the Appendices as follows:

The Diagram on the following page summarizes the relationship of the three methods.

It is quite clear from the chart that the isoperimetric profile plays a central role. In fact, one could state Theorems V and VI in terms of the isoperimetric profile and $\mathscr{R}_{\min } D^{2}(g)$ (the diameter appears as a scaling factor). The geometric assumption $r_{\min }(g) D^{2}(g) \geq(n-1) \varepsilon \alpha^{2}$ implies a good control on the isoperimetric profile (see Appendix I). Any improvement on the estimates on the isoperimetric constants or on the isoperimetric profile will lead to better estimating theorems.

G. From vanishing to estimating theorems: an account. The first steps towards answering Questions (Q1) and (Q2) in §E (using analytic methods) were given by P. $\mathrm{Li}([\mathbf{L i}], 1979)$, in the case of Betti numbers. The main ingredients in P. Li's paper are inequality (L) (Appendix II), Kato's inequality (K1) (§E), J. Moser's iteration method (Appendix V) and Sobolev inequalities. The main difficulty in applying J. Moser's method is to control the constants in the Sobolev inequalities, in terms of (a minimal set of) geometric data on $(M, g)$. In [Li], $\mathrm{P}$. Li makes use of an isoperimetric inequality due to $\mathrm{Ch}$. Croke ([Cr], using dimension, volume, diameter and Ricci curvature of $(M, g))$. P. Li's paper was later improved by S. Gallot ([Ga1-2], 1980-1981) 


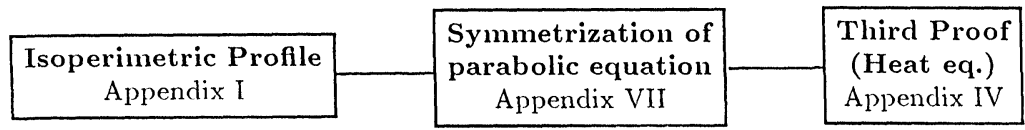

Let us summarize :

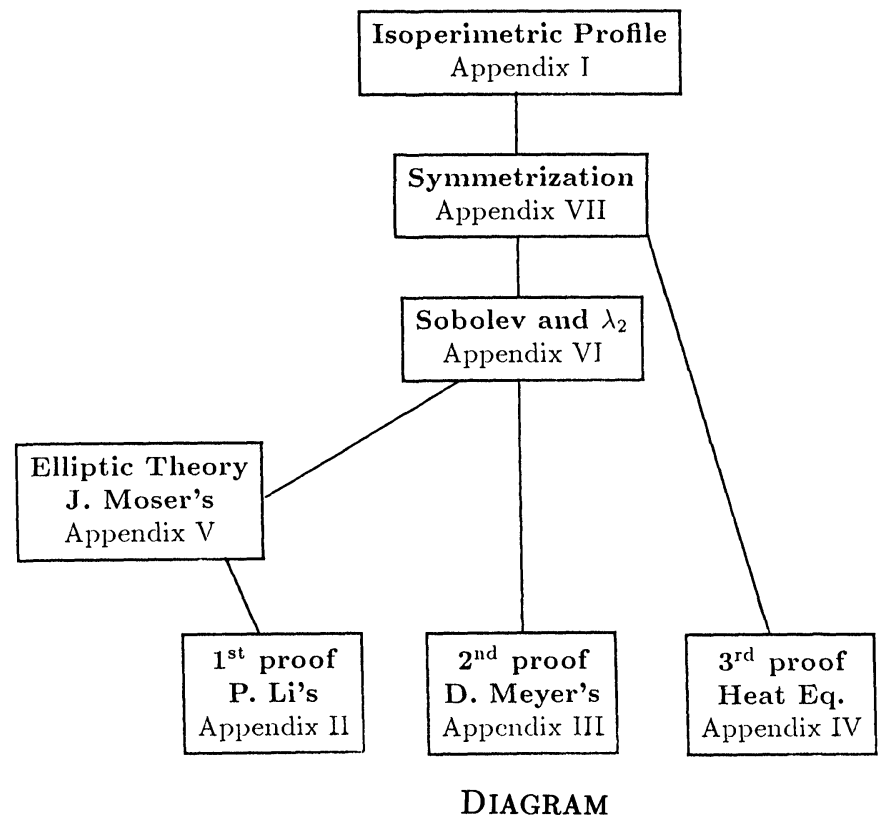

in two directions: the results are stated in a more general context; S. Gallot establishes new isoperimetric inequalities giving a qualitatively sharp control on the Sobolev constants. His final results depend on (dimension, diameter and Ricci curvature of $(M, g))$.

Since 1981, new analytic and isoperimetric methods have been developed. In [Bé-Ga], we initiated the heat equation method described in $\S \mathrm{F}$ and Appendices IV and VII. This was then improved in [Bé-Be-Ga1 and Bé, Chapter VI]. At the same time, following the lines of [Bé-Ga], the method of symmetrization of elliptic inequalities was applied in [Gam1] and more recently in [Gam2]: $\S \mathrm{F}$ and Appendices II and VII.

Quite recently, D. Meyer ([Me2], 1984) developed another method to answer Question (Q1), and possibly Question (Q2) ([Me3], 1986): see §F and Appendix III.

H. Possible extensions of the methods. In this paper, we only deal with closed Riemannian manifolds. Exactly the same methods would apply for compact Riemannian manifolds with boundary, provided Dirichlet conditions are assumed on the boundary (see [Bé-Ga, Bé-Be-Ga1]). For generalizations to the compact case (with boundary and Dirichlet or Neumann conditions on the boundary), we refer to [Co and Me2-3].

In $\S \mathrm{E}$, we gave examples in which one can apply the methods of this paper. Other examples include the following geometric or topological invariants: the 
dimension of the space of Killing or conformal vector-fields [Wu]; the $\widehat{A}$ genus, the dimension of the moduli space of an Einstein metric [Ga1-3, BéGa]; pinching and Betti numbers [Gam1, Hu]; minimal immersion into the sphere [El]; harmonic maps [Ad-Su, Ur]; pinching theorems [Ga1, Gam1, Bé-Be-Ga1]),...

For the complex case, we refer to the reviews $[\mathbf{S i 1}, \mathbf{2}, \mathbf{S h}-\mathbf{S o}, \mathbf{W u}]$ and to [De].

In some cases, one can extend the methods to noncompact manifolds [ $\mathbf{W u}$, Do].

Finally, we mention [Bé-Be] and the survey [Ro], where the estimates involve integral norms of the function $\mathscr{R}(x)$ instead of the $L^{\infty}$ norm $\mathscr{R}_{\text {min }}$ (see also Note added in proofs at the end of the paper).

I. Final comments. Theorems V and VI $(\S \mathrm{E})$ show that curvature estimates plus scaling imply global restrictions on the manifold $M$. One can also view these theorems as obstructions to the existence of special metrics on the manifold $M$ : this is a very important problem in geometry (see also the motivations given in the Introduction).

The methods which have been used in order to study this type of problem involve both geometric techniques and analytic techniques.

On the purely geometric side, one finds the classical comparison theorems (Bishop-Gromov, Rauch, Toponogov) and an extensive use of geodesics.

More recently, proofs have used minimal surfaces as a tool for proving geometric theorems (as a 2-dimensional generalization of geodesics, with much more analysis involved).

In many problems, a natural Weitzenböck formula plays a central role, and we in fact deal with an extension of the Bochner Technique; see $\S \mathrm{H}$.

In the diagram which appears on the next page, we list some geometric results on the structure of a Riemannian manifold, under a certain curvature assumption (1st column). Column 2 lists results obtained by a geometric method (which may involve analysis, as in the case in which minimal surfaces are used); column 3 lists results obtained by the Bochner technique. The comparison of columns 2 and 3 is quite instructive (compare with [Ka, Yau1,2]).

The assumption $r_{\min }(g) D^{2}(g) \geq(n-1) \varepsilon \alpha^{2}$ in $\S \mathrm{E}$, Theorems V, VI and Appendix $\mathrm{I}$ is quite natural in view of $\mathrm{M}$. Gromov's precompactness theorem (see [Sa or Bé, Chapter VI]). For counter-examples showing that $(n, \varepsilon, \alpha, D(g))$ is a minimal set of geometric assumptions in order to insure the validity of the theorems, we refer to [Bé, Be-Ga, Ga1-3].

As we already pointed out before, Theorems V and VI are quite effective in the sense that they actually permit explicit computations of the bounds. Numerical experiments [Bé-Ga, Gam1] seem to show that

(i) (Symmetrization + P. Li's inequality (L)) gives sharper results than the other methods when $\left|\mathscr{R}_{\text {min }} D^{2}(g)\right|$ is small; follow D. Meyer's method and the heat equation method;

(ii) Heat equation methods give sharper results than the other methods when $\left|\mathscr{R}_{\min } D^{2}(g)\right|$ is large; 


\begin{tabular}{|c|c|c|}
\hline \begin{tabular}{|l|l|} 
Curvature \\
Assumption
\end{tabular} & $\begin{array}{l}\text { Geometric (does not } \\
\text { exclude using analysis) }\end{array}$ & Bochner analytic \\
\hline $\begin{array}{l}\text { Positive curvature } \\
\text { operator }\end{array}$ & $\begin{array}{l}\text { Sphere theorem: } \\
\text { J. D. Moore [Mo]; } \\
\text { M. Micallef-J. D. Moore } \\
\text { [Mi-Mo] }\end{array}$ & $\begin{array}{l}\text { Vanishing theorems for } \\
\text { Betti numbers: } \\
\text { D. Meyer [Me1]; } \\
\text { S. Gallot and D. Meyer } \\
\text { [Ga-Me] }\end{array}$ \\
\hline curvature op. $\geq-a^{2}$ & & $\begin{array}{l}\text { Bounds on Betti numbers: } \\
\text { P. Li }[\mathbf{L i}] \text { and this survey }{ }^{2}\end{array}$ \\
\hline Sect. curv. pinching & $\begin{array}{l}\text { Sphere theorems: } \\
\text { [Che-Eb] and [Sa] }\end{array}$ & \\
\hline Sect. curv. $\geq-a^{2}$ & $\begin{array}{l}\text { Bounds on Betti numbers: } \\
\text { M. Gromov }[\mathbf{G r} \mathbf{1}]^{2}\end{array}$ & \\
\hline $\mid$ Sect. curv. $\mid \leq k$ & $\begin{array}{l}\text { Almost flat manifold theorem: } \\
\text { M. Gromov }[\mathbf{B u}-\mathbf{K a}]^{2} \text {; } \\
\text { compactness theorem: } \\
\text { M. Gromov }[\mathbf{G r 3}]^{3}\end{array}$ & $\begin{array}{l}\text { Bounds on Betti numbers: } \\
\text { P. Li [Li] and this survey }\end{array}$ \\
\hline Sect. curv. $\geq 0$ & $\begin{array}{l}\text { Soul theorem: J. Cheeger, } \\
\text { D. Gromoll, W. Meyer } \\
\text { [Che-Eb] }\end{array}$ & \\
\hline Ricci curv. $\geq-a^{2}$ & $\begin{array}{l}\text { Bound on 1st Betti number: } \\
\text { M. Gromov [Gr2 }]^{2} \text {; } \\
\text { precompactness theorem: } \\
\text { M. Gromov [Gr3 }]^{2} \text {; } \\
\text { isoperimetric estimator: } \\
\text { Appendix I in this survey } \\
\end{array}$ & $\begin{array}{l}\text { Bound on 1st Betti number: } \\
\text { P. Li [Li] and this survey }\end{array}$ \\
\hline Ricci curv. $\geq 0$ & $\begin{array}{l}\text { Structure theorems: } \\
\text { J. Cheeger-D. Gromoll, } \\
\text { J. Milnor [Che-Eb]; } \\
\text { M. Gromov [Gr3]; } \\
\text { R. Schoen and } \\
\text { S. T. Yau [Sc-Yau] }\end{array}$ & $\begin{array}{l}\text { "Vanishing" theorem for } \\
\text { 1st Betti number: } \\
\text { S. Bochner [Wu] }\end{array}$ \\
\hline Ricci curv. $>0$ & $\pi_{1}$ finite: S. Myers [Che-Eb] & $\begin{array}{l}\text { Vanishing theorem for } \\
\text { 1st Betti number: } \\
\text { S. Bochner [Wu] }\end{array}$ \\
\hline Scal. curv. $\geq 0$ & $\begin{array}{l}\text { Structure theorems and } \\
\text { positive energy: R. Schoen } \\
\text { and S. T. Yau [Ka] }\end{array}$ & $\begin{array}{l}\text { Vanishing theorem for } \\
\hat{A} \text {-genus: A. Lichnerowicz } \\
{[\mathbf{L z}] ; \text { Structure theorems }} \\
\text { and positive energy } \\
\text { M. Gromov and B. Lawson } \\
\text { [Gr-La]; E. Witten [Ka] }\end{array}$ \\
\hline
\end{tabular}

${ }^{2} D(g)$ bounded from above;

${ }^{3} D(g)$ bounded from above, $V(g)$ bounded from below. 
(iii) (J. Moser + Sobolev + P. Li) does not give the best numerical results, but sometimes works when the other methods fail [Gam1].

Appendix I: The isoperimetric profile. Let $(M, g)$ be a closed Riemannian manifold. An isoperimetric inequality on $(M, g)$ is an estimate from below of the $((n-1)$-dimensional) volume of the boundary $\partial \Omega$ of a smooth open submanifold $\Omega$, in terms of the ( $n$-dimensional) volume of $\Omega$. Since $\Omega$ and $M \backslash \Omega$ have the same boundary, we need consider relative volumes. We define the isoperimetric profile ("function" in [Bé]) $h(M, g ; s)$ of $(M, g)$ as:

$$
\begin{aligned}
h(M, g ; s) & =\inf \{\operatorname{vol}(\partial \Omega) / V(g): \Omega \subset M \\
& \text { smooth open submanifold such that } \operatorname{vol}(\Omega)=s V(g)\} .
\end{aligned}
$$

for $s \in[0,1](V(g)$ is the volume of $(M, g))$.

An isoperimetric estimator on $(M, g)$ is a function $H:[0,1] \rightarrow \mathbf{R}_{+}$such that $h(M, g ; s) \geq H(s)$ for all $s \in[0,1]$.

Classically, one defines the following isoperimetric constants on $(M, g)$ :

$$
\begin{gathered}
I(M, g)=\inf \left\{\operatorname{vol}(\partial \Omega) / \min [\operatorname{vol}(\Omega), \operatorname{vol}(M \backslash \Omega)]^{(n-1) / n}: \Omega \subset M\right\}, \\
h_{C}(M, g)=\inf \{\operatorname{vol}(\partial \Omega) / \min [\operatorname{vol}(\Omega), \operatorname{vol}(M \backslash \Omega)]: \Omega \subset M\},
\end{gathered}
$$

where the infimum is taken over all smooth open submanifolds of $(M, g)$. The constant $I(M, g)$ is called the isoperimetric constant of $(M, g)$, and $h_{C}(M, g)$ is known as J. Cheeger's isoperimetric constant. Clearly, one has the inequalities

$$
\begin{gathered}
I(M, g) V(g)^{1 / n}=\inf \left\{h(M, g ; s) / s^{(n-1) / n}: s \in[0,1 / 2]\right\} \\
h_{C}(M, g)=\inf \{h(M, g ; s) / s: s \in[0,1 / 2]\} .
\end{gathered}
$$

By (a), one has the isoperimetric estimator $H_{I}(s)$,

$$
H_{I}(s)=I(M, g) V(g)^{1 / n} \min [s, 1-s]^{(n-1) / n} .
$$

This estimator is used in [Bé-Ga and Gam1] with a "double-disk" as model space.

By (b), one has the isoperimetric estimator $H_{C}(s)$,

$$
H_{C}(s)=h_{C}(M, g) \min [s, 1-s] .
$$

This estimator is used in [Bé, Chapter IV] to give a proof à la Faber-Krahn of J. Cheeger's lower bound on the first nonzero eigenvalue of $(M, g)$.

The proofs given in Appendices VI and VII (Theorems VI.3 and VII.2) rely on the assumption that $(M, g)$ has an isoperimetric estimator of the form $H_{R}(s)=h\left(S^{n}(R)\right.$, can; $\left.s\right)$ for some positive number $R$, where $\left(S^{n}(R)\right.$, can) is the canonical sphere of radius $R$ in $\mathbf{R}^{n+1}$ (in fact, we could use other isoperimetric estimators, as explained in [Bé, Chapter IV]). Such an estimator (i.e. such an $R$ ) is provided by the following theorem [Bé-Be-Ga1].

2. THEOREM. Let $\left(M^{n} g\right)$ be a closed $C^{\infty}$ Riemannian manifold, such that $r_{\min }(g) D^{2}(g) \geq(n-1) \varepsilon \alpha^{2}$ where $\alpha \in \mathbf{R}_{+}$and $\varepsilon \in\{-1,0,1\}$. Then, for all $s \in[0,1]$,

$$
h(M, g ; s) \geq h\left(S^{n}(R), \operatorname{can} ; s\right)=R^{-1} h\left(S^{n}(1), \text { can; } s\right),
$$


where

$$
\begin{aligned}
& \bullet R=R(n, \varepsilon, \alpha ; D(g))=D(g) / a(n, \varepsilon, \alpha), \\
& \bullet a(n, \varepsilon, \alpha)= \begin{cases}\alpha \omega_{n}^{1 / n}\left(2 \int_{0}^{\alpha / 2} \cos ^{n-1} t d t\right)^{-1 / n} & \text { if } \varepsilon=1, \\
\left(1+n \omega_{n}\right)^{1 / n}-1 & \text { if } \varepsilon=0, \\
\alpha C(\alpha) & \text { if } \varepsilon=-1,\end{cases} \\
& \text { - } \omega_{n}=\int_{0}^{\pi} \sin ^{n-1} t d t, \\
& -C(\alpha) \text { is the unique positive root of the equation }
\end{aligned}
$$

$$
x \int_{0}^{\alpha}(c h t+x s h t)^{n-1} d t=\omega_{n} .
$$

REMARK. The assumptions in Theorem 2 are those of the pre-compactness theorem of M. Gromov (see [Sa or Bé, Chapter VI]). For some properties of $h(M, g ; s)$ we refer to [Ba-Pa].

A slight generalization of the notion of Schwarz symmetrization (see e.g. [Ba or Bé, Chapter IV]), and Theorem 2 will enable us to compare functions on $M$ to radial functions on $S^{n}$. This symmetrization preserves the $L^{p}$-norms of the functions, and decreases energies or more generally the $L^{p}$-norms of the gradients (up to normalizing constants). These two simple (and classical) facts are the key for estimating Sobolev constants on $(M, g)$ in terms of Sobolev constants on $S^{n}$ (Appendix VI), and for comparison of elliptic or parabolic inequalities (Appendix VII).

The underlying philosophy is that results such as Theorems V and VI $(\S \mathrm{E})$ should be stated as: one can bound $\delta(E)$ in terms of $\mathscr{R}_{\min } D^{2}(g)$ and a good isoperimetric estimator for $(M, g)$.

Appendix II: Using P. Li's lemma. The following general lemma has been used by $\mathrm{P}$. $\mathrm{Li}[\mathbf{L i}$, Lemma 11, p. 460] in the context of differential forms.

1. LEMMA. For a smooth section $s$ of the fiber bundle $E$ over the Riemannian manifold $(M, g)$, let

$$
\begin{gathered}
\|s\|_{\infty}=\sup \left\{|s|_{x}: x \in M\right\}, \quad\|s\|_{2}^{2}=\int_{M}|s|_{x}^{2} d v_{g}(x), \\
L(s)=\operatorname{vol}(M, g)\|s\|_{\infty}^{2} /\|s\|_{2}^{2} .
\end{gathered}
$$

Given a finite dimensional subspace $F$ of $C^{\infty}(E)$, we have

$$
\operatorname{dim} F \leq l \sup \{L(s): s \in F \backslash\{0\}\},
$$

where $l$ is the rank of $E$.

Proof. In this proof, orthonormal means orthonormal with respect to the $L^{2}(E)$-real inner product given by

$$
(s, t) \mapsto \int_{M}\langle s(x), t(x)\rangle_{x} d v_{g}(x) .
$$


Let $N=\operatorname{dim} F$ and let $\left\{e_{1}, \cdots, e_{N}\right\}$ be an orthonormal basis of $F$. Define a function $f$ on $M$ by

$$
f(x)=\sum_{i=1}^{N}\left|e_{i}(x)\right|^{2}
$$

The function $f$ does not depend on the choice of an orthonormal basis of $F$ : indeed, $f(x)$ is the trace of the kernel of the orthogonal projection onto $F$, evaluated at the point $(x, x)$.

Let $x_{0}$ be a point at which $f$ achieves its maximum. We can then write

$$
N=\int_{M} f(x) d v_{g}(x) \leq V(g) f\left(x_{0}\right) .
$$

Consider the linear map $S: F \rightarrow E_{x_{0}}$, defined by $S s=s\left(x_{0}\right)$. We can write $F$ as the following orthogonal sum: $F=\operatorname{Ker} S \oplus(\operatorname{Ker} S)^{\perp}$.

Take an orthonormal basis $\left\{f_{1}, \ldots, f_{N}\right\}$ of $F$, adapted to this decomposition of $F$, i.e. $\left\{f_{1}, \ldots, f_{m}\right\}$ is an orthonormal basis of $(\operatorname{Ker} S)^{\perp}$ and $\left\{f_{m+1}, \ldots, f_{N}\right\}$ is an orthonormal basis of $\operatorname{Ker} S$. Clearly, we have the inequality $m \leq \operatorname{rank}(S) \leq l=\operatorname{dim} E_{x_{0}}$. Using the invariance of $f$ referred to above, we can write

$$
f\left(x_{0}\right)=\sum_{i=1}^{m}\left|f_{i}\left(x_{0}\right)\right|^{2} \leq l \sup \left\{\|s\|_{\infty}^{2}: s \in F,\|s\|_{2}=1\right\} .
$$

The lemma follows immediately from this inequality and $(*)$.

In section (a) (resp. (b)), we use P. Li's lemma together with Sobolev inequalities (resp. symmetrization of elliptic inequalities) to answer Questions (Q1) and (Q2) of $\S \mathrm{E}$.

(a) P. Li's lemma and Sobolev inequalities. For $1 \leq q$, let $p$ satisfy $1 \leq p \leq n q /(n-q)$ and $p<\infty$. Define the Sobolev constant $\Sigma(n ; p, q)$ of the canonical unit sphere $S^{n}$ by

(a) $\Sigma(n ; p, q)=\sup \left\{\|f\|_{p} /\|d f\|_{q}: f \in W^{1, q}\left(S^{n}\right), f \neq 0, \int_{S^{n}} f=0\right\}$

(see $[$ Au 1, 2]).

For $n \geq 3$, define the constant $\sigma_{n}$ by

$$
\sigma_{n}=\Sigma(n ; 2 n /(n-2), 2) \operatorname{vol}\left(S^{n}\right)^{1 / n} .
$$

For $n=2$ and $1 \leq \nu<\infty$, define the constant $\sigma_{2, \nu}$ by

$$
\sigma_{2, \nu}=\Sigma(2 ; 2 \nu, 2) \operatorname{vol}\left(S^{n}\right)^{(\nu-1) / 2 \nu} .
$$

For $n \geq 2$ and $\nu \geq 1$, define the function $B: \mathbf{R}_{+} \rightarrow \mathbf{R}_{+}$by

$$
B(n, \nu ; x)=\prod_{i=0}^{\infty}\left(x \nu^{i}\left(2 \nu^{i}-1\right)^{-1 / 2}+1\right)^{2 \nu^{-i}} .
$$

(e) If the closed Riemannian manifold $\left(M^{n}, g\right)$ satisfies $r_{\min }(g) D^{2}(g) \geq$ $(n-1) \varepsilon \alpha^{2}$ then, by Appendix I, Theorem 2, there exists a positive number $R=D(g) / a(n, \varepsilon, \alpha)$ such that $h(M, g ; s) \geq h\left(S^{n}(R)\right.$, can; $\left.s\right)$ for any $s \in[0,1]$. 
By Appendix VI, Theorem 3, we then conclude that the following Sobolev inequalities hold:

$$
\left\{\begin{array}{l}
\text { For } n \geq 3 \\
\forall f \in W^{1, q}(M),\|f\|_{2 n /(n-2)} \leq V(g)^{-1 / n}\left[R \sigma_{n}\|d f\|_{2}+\|f\|_{2}\right] \\
\text { For } n=2 \text { and any } \nu \geq 1 \\
\forall f \in W^{1, q}(M),\|f\|_{2 \nu} \leq V(g)^{-(\nu-1) / 2 \nu}\left[R \sigma_{2, \nu}\|d f\|_{2}+\|f\|_{2}\right]
\end{array}\right.
$$

3. THEOREM. With the above notations and under the assumption

$$
r_{\min }(g) D^{2}(g) \geq(n-1) \varepsilon \alpha^{2},
$$

let $E(\lambda)$ denote the space of eigensections of $\widetilde{\Delta}$ associated with the eigenvalue $\lambda$. Then,

$$
\operatorname{dim} E(\lambda) \leq l B\left(n, \nu ; D(g)\left(\lambda-\mathscr{R}_{\min }\right)_{+}^{1 / 2} \sigma / a(n, \varepsilon, \alpha)\right)
$$

where

$$
\begin{cases}\nu \in\left[1, \infty\left[\text { and } \sigma=\sigma_{2, \nu},\right.\right. & \text { for } n=2 \\ \nu=n /(n-2) \text { and } \sigma=\sigma_{n}, & \text { for } n \geq 3\end{cases}
$$

REMARK. In particular, Theorem 3 gives an upper bound for $\delta(E)=$ $\operatorname{dim} E(\lambda)$, as stated in $\S \mathrm{E}$, Theorem VI.

PROOF OF THEOREM 3. Multiplying the Weitzenböck formula (W) by $s$, we can write (see $\S \mathrm{E}$ )

$$
\langle\widetilde{\Delta} s, s\rangle=\langle\bar{\Delta} s, s\rangle+\langle\mathscr{R} s, s\rangle=\frac{1}{2} \Delta(\langle s, s\rangle)+|D s|^{2}+\langle\mathscr{R} s, s\rangle .
$$

Using the second Kato inequality (K2) of $\S \mathrm{E}$, we obtain the following distributional inequality:

$$
\Delta(|s|) \leq\left(\lambda-\mathscr{R}_{\min }\right)|s|
$$

for any $s \in E(\lambda)$.

Taking $f=|s|$ and $a=\left(\lambda-\mathscr{R}_{\text {min }}\right)_{+}$, we can apply Appendix V, Theorem 3 (J. Moser's iterative method) with $\delta=1$ and $\gamma=R \sigma_{n} V(g)^{-1 / n}$ (for $n \geq 3$ ) or $\gamma=R \sigma_{2, \nu} V(g)^{-(\nu-1) / 2 \nu}$ (for $n=2$ ). The result follows from inequality (L) and Theorem V.3.

5. COROLLARY. In the preceding context, there exists a number $b(n, \varepsilon, \alpha)$, such that $\left(D^{2}(g) \mathscr{R}_{\min }\right)_{-} \leq b(n, \varepsilon, \alpha)$ implies $\delta(E) \leq l$.

Proof. Use the fact that $\lim _{x \rightarrow 0_{+}} B(n, \nu ; x)=1$, and observe that $\delta(E)<$ $l+1 \Rightarrow \delta(E) \leq l$.

6. THEOREM. Let $\bar{N}_{E}(\lambda)$ be the counting function for the eigenvalues of the rough Laplacian $\bar{\Delta}$ acting on $C^{\infty}(E)$, i.e.

$$
\bar{N}_{E}(\lambda)=\#\left\{j: \bar{\lambda}_{j} \leq \lambda\right\}
$$

where $\bar{\lambda}_{1} \leq \bar{\lambda}_{2} \leq \cdots \nearrow+\infty$ are the eigenvalues of $\bar{\Delta}$. Then, $\bar{N}_{E}(\lambda) \leq$ $B\left(n, \nu ; R \lambda^{1 / 2} \sigma\right)$, with

$$
\begin{cases}\sigma=\sigma_{n}, \nu=n /(n-2), & \text { if } n \geq 3 \\ \sigma=\sigma_{2, \nu}, \nu \in[1, \infty[, & \text { if } n=2\end{cases}
$$


PROOF. Let $\mathscr{E}$ be the direct sum of the eigenspaces of $\bar{\Delta}$, corresponding to eigenvalues less than or equal to $\lambda$. One cannot apply directly the proof we gave for Theorem 3 , because inequality (4) does not hold for any $s \in \mathscr{E}$.

For $k \geq 2$, let $S_{k}=\sup \left\{\|s\|_{k} /\|s\|_{2}: s \in \mathscr{E} \backslash\{0\}\right\}$. By P. Li's lemma $\bar{N}_{E}(\lambda)=\operatorname{dim} \mathscr{E} \leq S_{\infty}^{2} V(g)$. It therefore suffices to bound $S_{\infty}$. Let $\left\{s_{i}\right\}$ be an orthonormal basis of $\mathscr{E}$, given by eigensections of $\bar{\Delta}, \bar{\Delta} s_{i}=\bar{\lambda}_{i} s_{i}$. For any $s \in \mathscr{E}$, we write $s=\sum_{i=1}^{N} a_{i} s_{i}$, where $N=\bar{N}_{E}(\lambda)=\operatorname{dim} \mathscr{E}$, and hence $\bar{\Delta} s=\sum_{i=1}^{N} \bar{\lambda}_{i} a_{i} s_{i}$. The map $[0, \lambda]^{N} \ni\left(\mu_{1}, \ldots, \mu_{N}\right) \mapsto\left\|\sum_{i=1}^{N} \mu_{i} a_{i} s_{i}\right\|_{k}$ being convex, its supremum is achieved on one of the vertices of the cube $[0, \lambda]^{N}$, so that we can write

$$
\|\bar{\Delta} s\|_{k} \leq \lambda\left\|\sum_{i \in I} a_{i} s_{i}\right\|_{k}
$$

where $I$ is a subset of $\{1,2, \ldots, N\}$. By definition of $S_{k}$,we have

$$
\|\bar{\Delta} s\|_{k} \leq \lambda S_{k}\left\|\sum_{i \in I} a_{i} s_{i}\right\|_{2} \leq \lambda S_{k}\|s\|_{2} .
$$

As in the proof of Theorem 3, we have the distributional inequality

$$
\Delta(|s|) \leq|\bar{\Delta} s| .
$$

Multiplying this inequality by $|s|^{2 k-1}$, integrating and applying Hölder's inequality, we obtain

$$
\left\|d|s|^{k}\right\|_{2}^{2} \leq k^{2}(2 k-1)^{-1}\|s\|_{2 k}^{2 k-1}\|\bar{\Delta} s\|_{2 k},
$$

i.e.

$$
\left\|d|s|^{k}\right\|_{2} \leq k(2 k-1)^{-1 / 2} \lambda^{1 / 2} S_{2 k}^{k}\|s\|_{2}^{k} .
$$

Assuming that $(M, g)$ satisfies the Sobolev inequality (1), we obtain

$$
S_{2 k n /(n-2)} \leq\left(R \lambda^{1 / 2} \sigma_{n} k(2 k-1)^{-1 / 2}+1\right)^{1 / k} V(g)^{-1 / n k} S_{2 k},
$$

for $n \geq 3$, and

$$
S_{2 k \nu} \leq\left(R \lambda^{1 / 2} \sigma_{2, \nu} k(2 k-1)^{-1 / 2}+1\right)^{1 / k} V(g)^{-(\nu-1) / 2 k \nu} S_{2 k},
$$

for $n=2$ and any $\nu \geq 1$.

Taking $k=(n /(n-2))^{i}, i \in \mathbf{N}$ when $n \geq 3$, or $k=\nu^{i}, i \in \mathbf{N}$ when $n=2$ (see Appendix V), we obtain an upper bound for $S_{\infty}$. It then suffices to apply inequality $(\mathrm{L})$ as we already mentioned above.

REMARK. From Theorem 6 and Appendices I and V, one can deduce that the eigenvalues $\bar{\lambda}_{i}$ of $\bar{\Delta}$ (this includes the usual Laplacian $\Delta$ on functions) can be bounded from below as follows:

$$
\bar{\lambda}_{i} D^{2}(g) \geq C\left(n, r_{\min }(g) D^{2}(g)\right) i^{2 / n},
$$

for some constant $C$, for $i$ large enough, and for $n \geq 3$. For $n=2$, one also obtains an estimate, but it is not optimal, we find $i^{(\nu-1) / \nu}$ instead of $i$. 
(b) P. Li's lemma and symmetrization of elliptic inequalities. The proofs of Theorems 3 and 6 rely on inequality $(L)$, i.e. on an estimate of $V(g)\|s\|_{\infty}^{2} /\|s\|_{2}^{2}$ for $C^{\infty}$ sections which satisfy a (distributional) inequality of the form $\Delta|s| \leq a|s|$, for some real number $a$. In section (a), we applied J. Moser's iteration scheme (Appendix V) and the estimates on the Sobolev constants given in Appendix VI. One can directly study the elliptic inequality $\Delta|s| \leq a|s|$, by using a symmetrization method. This is done in Appendix VII, where the following theorem is proved.

7. THEOREM. If the $C^{\infty}$ section $s$ of $E$ satisfies the distributional inequality $\Delta|s| \leq a|s|, a \in \mathbf{R}_{+}$, and if $(M, g)$ satisfies $r_{\min }(g) D^{2}(g) \geq$ $(n-1) \varepsilon \alpha^{2}$, we have

$$
\|s\|_{\infty}^{2} V(g) /\|s\|_{2}^{2} \leq \operatorname{vol}\left(S^{n}\right)\left[\operatorname{vol}\left(S^{n-1}\right) \int_{0}^{r_{b}} \varphi_{b}^{2}(r) \sin ^{n-1} r d r\right]^{-1}
$$

where $b=a D^{2}(g) / a^{2}(n, \varepsilon, \alpha)$ (see Appendix $\left.\mathrm{I}\right)$, where $\varphi_{b}$ is the regular solution of the differential equation

$$
y^{\prime \prime}(r)+(n-1) \operatorname{cotg} r y^{\prime}(r)+b y(r)=0 \quad \text { on }[0, \pi[
$$

which satisfies $\varphi_{b}(0)=1$, and where $r_{b}$ is the first positive zero of $\varphi_{b}$.

This theorem provides another proof of Theorem 3, using P. Li's lemma. It is easy to see that the right-hand side of the inequality in Theorem 7 goes to 1 when $b$ goes to zero, so that Theorem 7 also implies Corollary 5 . Numerical experiments [Gam1] show that Theorem 7 gives sharper results than Theorem 3 and Corollary 5 (the same isoperimetric inequalities were used to derive both Theorem 7 and Theorem 3).

Appendix III: Application of Daniel Meyer's lemma. In [Me2], Daniel Meyer introduced the following lemma which is somehow the dual version of $\mathrm{P}$. Li's inequality (L).

LEMMA. Let $F$ be a finite dimensional subspace of the space $L^{2}(E)$ of $L^{2}$-sections of the Riemannian fiber bundle $E$ over the closed Riemannian manifold $(M, g)$. Assume that the dimension $N$ of $F$ is larger than the rank $l$ of $E$. Then there exists a section $s$ in $F$ such that

$$
\|s\|_{1} \leq C(N, l) V(g)^{1 / 2}\|s\|_{2},
$$

where the function $C(N, l)$ is given by

$$
C(N, l)=(N / l)^{1 / 2} \Gamma((l+1) / 2) \Gamma(N / 2) / \Gamma(l / 2) \Gamma((N+1) / 2)
$$

$(\Gamma(x)$ is the usual gamma function). In particular, $C(N, N)=1$ and the function $N \mapsto C(N, l)$ is a (strictly) decreasing function of $N$.

The proof of this lemma is quite intricate, we refer to [Me2].

COMMENTS. In the proof of S. Bochner's theorem ( $\S \mathrm{D})$, we used the fact that the vector space of parallel sections $(D s=0)$ of $E$ has dimension less than or equal to the rank $l$ of $E$. From the equality $d|s|^{2}=2\langle D s, s\rangle$, we deduce that the point-wise norm $|s|_{x}$ of a parallel section is constant on $M$. 
P. Li's inequality (L) (Appendix II) shows that if $F$ is a finite dimensional subspace of $C^{\infty}(E)$, whose elements have constant norms, then $\operatorname{dim} F$ is less than or equal to $l$. In fact, the same is true if any element $s$ in $F$ has "almost constant" norm in the sense that $\|s\|_{\infty}^{2} V(g)\|s\|_{2}^{-2}<(l+1) / l$. This is exactly how we used (L) in Appendix II. On the other hand, Daniel Meyer's lemma shows that if $\operatorname{dim} F$ is bigger than $l$, then there exists a section $s$ in $F$ whose norm is "far from being constant", in the sense that the function $|s|$ is not too close to its mean value over $M$ : indeed, for the section $s$ given by the lemma we have

$$
\left\||s|-V(g)^{-1} \int_{M}|s| v_{g}\right\|_{2}^{2} \geq\left[C(N, l)^{-1}-1\right] V(g)^{-1}\|s\|_{1}^{2} .
$$

We now show how one can answer Question (Q1) of $\S E$ by making use of D. Meyer's lemma.

Let us assume that $F=\left\{s \in C^{\infty}(E): \widetilde{\Delta} s=0\right\}$ has dimension at least $l+1$. Multiplying the Weitzenböck formula (W) by $s$ and integrating over $M$, gives

$$
0=\int_{M}\left(|D s|^{2}+\langle\mathscr{R} s, s\rangle\right) v_{g} \geq \int_{M}\left(|D s|^{2}+\mathscr{R}_{\min }|s|^{2}\right) v_{g}
$$

Applying Kato's inequality (K1) gives:

$$
\int_{M}|d| s||^{2} v_{g} \leq \int_{M}|D s|^{2} v_{g} \leq\left(-\mathscr{R}_{\min }\right) \int_{M}|s|^{2} v_{g} .
$$

For $s$ as given by the above lemma, let $f=|s|$ and $h=f-V(g)^{-1} \int_{M} f v_{g}$. For $f$, we can write $(N=\operatorname{dim} F \geq l+1)$ :

$$
\left(\int_{M} f v_{g}\right)^{2} \leq C^{2}(N, l) V(g) \int_{M} f^{2} v_{g}
$$

and hence

$$
\int_{M} h^{2} v_{g} \geq\left(1-C^{2}(l+1, l)\right) \int_{M} f^{2} v_{g}
$$

Since $d f=d h$, we can use (1) and conclude that

$$
\int_{M}|d h|^{2} v_{g} \leq\left(-\mathscr{R}_{\min }\right)\left(1-C^{2}(l+1, l)\right)^{-1} \int_{M} h^{2} v_{g}
$$

Recalling that $\int_{M} h v_{g}=0$, and using the variational characterization of the first nonzero eigenvalue $\lambda_{2}(M, g)$ of $(M, g)$, we obtain

$$
\lambda_{2}(M, g) \leq\left(-\mathscr{R}_{\min }\right)\left(1-C^{2}(l+1, l)\right)^{-1} .
$$

Under the assumption that $(M, g)$ satisifes

$$
r_{\min }(g) D^{2}(g) \geq(n-1) \varepsilon \alpha^{2} \quad \text { (see Theorem } 2 \text { in Appendix I) }
$$

we have the following lower bound for $\lambda_{2}(M, g)$ (Appendix VI, Corollary 8): $\lambda_{2}(M, g) \geq n a^{2}(n, \varepsilon, \alpha) / D^{2}(g)$, and hence finally

$$
n a^{2}\left(n, \varepsilon, \alpha^{2}\right)\left(1-C^{2}(l+1, l)\right) \leq-\mathscr{R}_{\min } D^{2}(g) .
$$


We have proved the

3. Proposition. Assume that the closed Riemannian manifold $\left(M^{n}, g\right)$ satisfies $r_{\min }(g) D^{2}(g) \geq(n-1) \varepsilon \alpha^{2}$. Then there exists a number $b(n, \varepsilon, \alpha)$ such that the inequality $-\mathscr{R}_{\min } D^{2}(g) \leq b(n, \varepsilon, \alpha)$ implies that $\delta(E) \leq l(\delta(E)=$ $\left.\operatorname{dim}\left\{s \in C^{\infty}(E): \widetilde{\Delta} s=0\right\}\right)$.

The basic point for Proposition 3 is inequality (2) which shows that any lower bound for $\lambda_{2}(M, g)$ (e.g. J. Cheeger's lower bound) will give a version of Proposition 3. Theorem 2 of Appendix I gives a very effective method for actually computing $b(n, \varepsilon, \alpha)$.

D. Meyer's lemma, as stated above, does not give an immediate answer to Question (Q2) of $\S \mathrm{E}$. However, D. Meyer has informed me that one can improve the lemma in order to apply it to answer Question (Q2) (see [Me3]). It should also be pointed out that D. Meyer's lemma applies in a very general context, e.g. if $M$ has a boundary (see [Me2] for applications in this direction).

Appendix IV: Using the heat equation. In this appendix, we apply Kato's inequality for the heat kernel of the rough Laplacian (Theorem 7 below), and the isoperimetric inequality for the heat kernel on functions (Theorem 8) to answer the Questions (Q1), (Q2) raised in $\S \mathrm{E}$.

Recall from $\S \mathrm{E}$ that we are given a Laplacian $\widetilde{\Delta}$ acting on the $C^{\infty}$ sections of $E ; \widetilde{\Delta}$ satisfies the Weitzenböck formula $(\mathrm{W}): \widetilde{\Delta}=\bar{\Delta}+\mathscr{R}$, where $\bar{\Delta}$ is the rough Laplacian associated with the quadratic form $\int_{M}|D s|^{2} v_{g}$. We denote by $\left\{\tilde{\lambda}_{i}\right\}_{i \geq 1}$ (resp. $\left\{\bar{\lambda}_{i}\right\}_{i \geq 1}$ ) the nondecreasing sequence of the eigenvalues of $\widetilde{\Delta}(\operatorname{resp} . \bar{\Delta})$ counted with multiplicities.

Integrating the Weitzenböck formula (W), we obtain

$$
\int_{M}\langle\widetilde{\Delta} s, s\rangle v_{g}=\int_{M}\left(|D s|^{2}+\langle\mathscr{R} s, s\rangle\right) v_{g} \geq \int_{M}\left(|D s|^{2}+\mathscr{R}_{\min }|s|^{2}\right) v_{g}
$$

The variational characterization of the eigenvalues and (1) give

$$
\tilde{\lambda}_{i} \geq \bar{\lambda}_{i}+\mathscr{R}_{\min }, \quad \text { for all } i \geq 1 .
$$

It is clear that $\inf \left\{\int_{M}|D s|^{2} v_{g}: \int_{M}|s|^{2} v_{g}=1\right\}=\bar{\lambda}_{1} \geq 0$; furthermore, if $\bar{\lambda}_{1}=0$, then the associated eigenspace consists of parallel sections $(D s=0)$, and hence has dimension at most $l$ :

$$
\text { If } \bar{\lambda}_{1}=0, \quad \text { multiplicity }\left(\bar{\lambda}_{1}\right) \leq l
$$

(compare with $\S \mathrm{D})$.

We can now use (2) and (3) to give a simple proof of S. Bochner's theorem (§B, Theorem II).

Define the partition functions

$$
\left\{\begin{array}{l}
\widetilde{Z}(t)=\sum_{i=1}^{\infty} \exp \left(-\tilde{\lambda}_{i} t\right)=\text { Trace } \exp (-t \widetilde{\Delta}) \\
\bar{Z}(t)=\sum_{i=1}^{\infty} \exp \left(-\bar{\lambda}_{i} t\right)=\text { Trace } \exp (-t \bar{\Delta}) .
\end{array}\right.
$$


By (3), we have that

$$
\lim _{t \rightarrow \infty} \bar{Z}(t) \leq l .
$$

In order to prove S. Bochner's theorem, write

$$
\begin{aligned}
\delta(E) & =\operatorname{dim}\left\{s \in C^{\infty}(E): \widetilde{\Delta} s=0\right\} \\
& =\text { mult. of } 0 \text { as eigenvalue of } \widetilde{\Delta} \text { (possibly } 0) \\
& \leq \widetilde{Z}(t), \quad \text { for any } t>0 .
\end{aligned}
$$

Using (2), we can also write

$$
\delta(E) \leq \exp \left(-\mathscr{R}_{\min } t\right) \bar{Z}(t), \quad \text { for any } t>0 .
$$

If $\mathscr{R}_{\min } \geq 0,(5)$ gives $\delta(E) \leq \lim _{t \rightarrow \infty} \exp \left(-\mathscr{R}_{\min } t\right) \bar{Z}(t) \leq l$. If $\mathscr{R}_{\min }>0$, we obtain similarly $\delta(E) \leq 0$.

Notice that if $\mathscr{R}_{\min } \geq 0$ and $\mathscr{R}\left(x_{0}\right)>0$ at some point $x_{0}$ in $M,(1)$ implies that $\tilde{\lambda}_{1}>\bar{\lambda}_{1} \geq 0$, and hence we conclude as above that $\delta(E)=0$.

As was first pointed out by M. Gromov (private communication), the inequality $\delta(E) \leq \widetilde{Z}(t)$ suggests that one should be able to bound $\delta(E)$ by using heat equation techniques. This is indeed true, as was shown in [Bé-Ga] (see also [Bé, Chapter VI]). For this purpose, one needs two ingredients: Kato's inequality (Theorem 7 below) which bounds $\bar{Z}(t)$ from above by $l$ times the partition function $Z(t)$ of $(M, g)$ and Theorem 8 , which bounds $Z(t)$ from above under certain geometric assumptions.

Denote by $\left\{\lambda_{i}(M, g)\right\}_{i \geq 1}$ the nondecreasing sequence of the eigenvalues of $(M, g)$ for the Laplacian acting on functions. Define the partition function $Z(M, g ; t)$ by

$$
Z(M, g ; t)=\sum_{j=1}^{\infty} \exp \left(-\lambda_{j}(M, g) t\right) .
$$

For the following theorem, we refer to $[\mathbf{H}-\mathbf{S}-\mathbf{U}]$ or [Bé, Chapter VI and Appendix A].

7. THEOREM. Let $\bar{Z}(t)$ be the partition function for the rough Laplacian acting on $C^{\infty}$ sections of a Riemannian vector bundle $E$ of rank $l$ over the closed Riemannian manifold $(M, g)$ (formulas (4) and (6) supra). Then,

$$
\bar{Z}(t) \leq l Z(M, g ; t) .
$$

Note that Theorem 7 is best possible, because equality holds for $E=$ $M \times \mathbf{R}^{l}$, the trivial bundle.

From Theorem 7 and Appendix VII, Theorem 7, we finally deduce the

8. THEOREM. Let $E$ be a Riemannian vector bundle of rank $l$ over a closed Riemannian manifold $\left(M^{n}, g\right)$ which satisfies $r_{\min }(g) D^{2}(g) \quad \geq$ $(n-1) \varepsilon \alpha^{2}$ (see Appendix I). For the Laplacian $\widetilde{\Delta}=\bar{\Delta}+\mathscr{R}$ acting on $C^{\infty}(E)$, one has the inequalities

$$
\widetilde{Z}(t) \leq l \exp \left(-\mathscr{R}_{\min } t\right) Z\left(S^{n}, \operatorname{can} ; a^{2}(n, \varepsilon, \alpha) / D^{2}(g)\right),
$$


where $a(n, \varepsilon, \alpha)$ is defined in Appendix I, Theorem 2. In particular

$$
\delta(E)=\operatorname{dim} \operatorname{Ker} \widetilde{\Delta} \leq l \inf _{t>0} F(t),
$$

where $F(t)=\exp \left(-\mathscr{R}_{\min } D^{2}(g) t\right) Z\left(S^{n}, \operatorname{can} ; t a^{2}(n, \varepsilon, \alpha)\right)$.

CONSEquences. (i) Take $t=1$ in (9): this gives an upper bound on $\delta(E)$, involving $n, \varepsilon, \alpha$ and $\mathscr{R}_{\min } D^{2}(g)$;

(ii) Take $\varepsilon=-1, \alpha=1$ (by scaling) and minimize the RHS of (9) to get a number $\eta$ such that $-\mathscr{R}_{\min } D^{2}(g)<\eta$ implies $\delta(E) \leq l$;

(iii) Take $E=M \times \mathbf{R}, \widetilde{\Delta}=\Delta$ and get an upper bound for $N(\lambda)=$ $\#\left\{j: \lambda_{j}(M, g) \leq \lambda\right\}$, namely

$$
N(\lambda) \leq e Z\left(S^{n}, \operatorname{can} ; a^{2}(n, \varepsilon, \alpha) / \lambda D^{2}(g)\right)
$$

(iv) Take $E=T^{*} M, \widetilde{\Delta}=$ Hodge-de Rham Laplacian on 1-forms and get bounds on $b_{1}(M)$ in terms of $n, \varepsilon, \alpha$;

(v) Take $E=\bigwedge^{p} T^{*} M, \widetilde{\Delta}=$ Hodge-de Rham Laplacian on $p$-forms and get bounds on $b_{p}(M)$ in terms of $n, \varepsilon, \alpha$ and $K D^{2}(g)$ where $K$ is an upper bound for the absolute value of the sectional curvature of $(M, g)$; see [Bé-Ga];

(vi) Take $\lim _{t \rightarrow \infty} \log (Z(t)-1)$, and get another proof of Appendix VI, Corollary 8. This improves the classical Lichnerowicz-Obata theorem [B-GM, Bé-Me];

(vii) Using the fact that $Z\left(S^{n}\right.$, can; $\left.t\right) \leq A_{n} t^{-n / 2}+B_{n}$, for some positive constants $A_{n}, B_{n}$, one can deduce from Theorem 8 and (iii) lower bounds on the eigenvalues of $\Delta$, of the form

$$
\lambda_{i} D^{2}(g) \geq C(n, \varepsilon, \alpha) i^{2 / n}, \quad \text { for } n \geq 2 \text { and } i \geq 2
$$

(compare with the last remark in Appendix II, part (a); for references on such bounds, see [Ch, Chapter III, XII; Bé-Ber, Chapter 11; Bé, Chapter V].

Appendix V: J. Moser's iteration scheme. Let $(M, g)$ be an $n$-dimensional closed $C^{\infty}$ Riemannian manifold.

Assume that $(M, g)$ satisfies the following Sobolev inequality (recall that $V(g)=\operatorname{vol}(M, g))$ :

(1) For any $f \in W^{1,2}(M), \quad\|f\|_{2 \nu} \leq \gamma\|d f\|_{2}+\delta V(g)^{-(\nu-1) / 2 \nu}\|f\|_{2}$,

for some positive constants $\gamma$ and $\delta$, where $\nu$ is any number in $[1, \infty[$ if $n=2$ and $\nu=n /(n-2)$ when $n \geq 3$ (in that case, $n=2 \nu /(\nu-1)$ ). Notice that Hölder's inequality implies that $\delta \geq 1$. Define $B=B_{n, \delta}: \mathbf{R}_{+} \rightarrow \mathbf{R}_{+}$by

$$
B_{n, \delta}(x)=\prod_{i=0}^{\infty}\left(x \nu^{i}\left(2 \nu^{i}-1\right)^{-1 / 2}+\delta\right)^{2 \nu^{-i}}
$$

for $n \geq 2$ and $\delta \in \mathbf{R}_{+}$.

3. THEOREM. Let $f \in W^{1,2}(M)$ be a nonnegative continuous function such that $\Delta f \leq a f$ in the sense of distributions, for some real number $a$. Then, if $a<0, f$ must vanish identically. If $a \geq 0$, and if $(M, g)$ satisfies the Sobolev inequality (1) then

$$
\|f\|_{\infty}^{2} V(g) /\|f\|_{2}^{2} \leq B_{n, \delta}\left(\gamma V(g)^{(\nu-1) / 2 \nu} a^{1 / 2}\right),
$$


where $\nu \in[1, \infty[$ if $n=2$, and $\nu=n /(n-2)$ if $n \geq 3$. If $\Delta f=a f$, one can remove the assumption $f \geq 0$.

REMARKS. (i) The method of proof is due to J. Moser (see [Gi-Tr, p. 180]);

(ii) The assumption that $f$ is continuous is not really necessary; it is satisfied in the context in which we apply the theorem (Appendix II, part (a)) and simplifies the proof;

(iii) Recall that our Laplacian is $\Delta f=-f^{\prime \prime}$ on $\mathbf{R}$;

(iv) The best results are obtained when $\delta=1$ and $n \geq 3$. Indeed, when $n \geq 3$, we can take for $\nu$ the limit value $n /(n-2)$. In the case $n=2$, we cannot take $\nu=\infty$ because $W^{1,2}$ does not embed in $L^{\infty}$ [Au1, Chapter 2];

(v) We give the proof of Theorem 3 for completeness (it is very simple), and because we use it in the proof of Theorem 6 in Appendix II.

PROOF OF THEOREM 3. If $a<0$, multiplying the inequality $\Delta f \leq a f$ by $f$ and integrating over $M$ gives $\int_{M}|d f|^{2} v_{g} \leq a \int_{M} f^{2} v_{g}$. It follows that $f$ vanishes identically. For $a \geq 0$, multiply the inequality $\Delta f \leq a f$ by $f^{2 k-1}$ $(k \geq 1)$, and integrate. An elementary computation gives

$$
\left\|d\left(f^{k}\right)\right\|_{2} \leq a^{1 / 2} k(2 k-1)^{-1 / 2}\|f\|_{2 k}^{k} .
$$

Let $\mu=(\nu-1) / 2 \nu$. Apply Sobolev inequality (1) to $f^{k}$, using (4); this gives

$$
\left\|f^{k}\right\|_{2 \nu} \leq \gamma a^{1 / 2} k(2 k-1)^{-1 / 2}\|f\|_{2 k}^{k}+\delta V(g)^{-\mu}\left\|f^{k}\right\|_{2},
$$

and

$$
\|f\|_{2 k \nu} \leq\left(\gamma V(g)^{\mu} a^{1 / 2} k(2 k-1)^{-1 / 2}+\delta\right)^{1 / k} V(g)^{-\mu / k}\|f\|_{2 k} .
$$

Write inequality (5) for $k=\nu^{i}, i=0,1,2, \ldots$, and multiply these inequalities to obtain

$$
\|f\|_{\infty}^{2} \leq \prod_{i=0}^{\infty}\left[x \nu^{i}\left(2 \nu^{i}-1\right)^{-1 / 2}+\delta\right]^{2 \nu^{-i}} V(g)^{-1}\|f\|_{2}^{2}
$$

(we have used the fact that $\lim _{p \rightarrow \infty}\|f\|_{p}=\|f\|_{\infty}$ ), where $x=\gamma V(g)^{\mu} a^{1 / 2}$, $\mu=(\nu-1) / 2 \nu$.

The following proposition gives the behaviour of $B_{n}=B_{n, 1}$ when $x$ goes to zero or to infinity. Clearly enough, it also describes the behaviour of $B_{n, \delta}$.

6. Proposition. The function $B_{n}=B_{n, 1}$ satisfies the inequalities

$$
B_{n}(x) \leq \begin{cases}\exp (2 x \sqrt{\nu} /(\sqrt{\nu}-1)), & \text { when } 0 \leq x \leq 1, \\ B_{n}(1) x^{2 \nu /(\nu-1)}, & \text { when } x \geq 1\end{cases}
$$

In particular, $\lim _{x \rightarrow 0_{+}} B_{n}(x)=1$, and $B_{n}(x) \leq B_{n}(1) x^{n}$ for $x \geq 1$, when $n \geq 3$.

PROOF. The second inequality is obvious. In order to prove the first one, notice that

$$
\begin{aligned}
\log B_{n}(x) & =\sum_{i=0}^{\infty} 2 \nu^{-i} \log \left(1+x \nu^{i}\left(2 \nu^{i}-1\right)^{-1 / 2}\right) \\
& \leq 2 x \sum_{i=0}^{\infty} \nu^{-i / 2}=2 x \sqrt{\nu} /(\sqrt{\nu}-1) .
\end{aligned}
$$


Appendix VI: Sobolev embedding constants. Let $(M, g)$ be an $n$ dimensional closed $C^{\infty}$ Riemannian manifold with volume $V(g)$. The Sobolev embedding theorem states that the Sobolev space $W^{1, q}(M)$, of functions whose derivatives of order at most 1 are in $L^{q}$, embeds continuously in $L^{p}(M)$, for $p$ satisfying $1 \leq p \leq n q /(n-q)$ and $p<\infty$. In particular, there exist constants $A_{p, q}$ and $B_{p, q}$ such that

$$
\forall f \in W^{1, q}, \quad\|f\|_{q} \leq A_{p, q}\|d f\|_{q}+B_{p, q}\|f\|_{q} .
$$

Indeed, it suffices to cover the manifold $M$ by charts and to apply the classical Sobolev embedding theorem in $\mathbf{R}^{n}$, which turns out to be easy because $M$ is compact [Au1, Theorem 2.20, p. 44].

It is not clear then how the Sobolev constants depend on the geometry of $(M, g)$. On the other hand, in many geometric problems, it is quite important to control the Sobolev constants in terms of the geometry of $(M, g)$; see Appendix II and [Au1].

In this appendix, we show that in order to give upper bounds on the Sobolev constants, it suffices to have a good isoperimetric estimator (see Appendix I). Such an estimator is given by Appendix I, Theorem 2, in terms of $\operatorname{dim} M$, a lower bound on the Ricci curvature of $(M, g)$ and an upper bound on the diameter of $(M, g)$.

We end this appendix with some comments on other methods which were used to estimate the Sobolev constants.

For $1 \leq p \leq n q /(n-q)$, and $p<\infty$, let $\Sigma(n ; p, q)$ be the Sobolev constant of the canonical unit sphere $S^{n}$, defined by

$$
\Sigma(n ; p, q)=\sup \left\{\|f\|_{p} /\|d f\|_{q}: f \in W^{1, q}\left(S^{n}\right), f \neq 0, \int_{S^{n}} f=0\right\} .
$$

3. THEOREM. Assume that there exists a positive number $R$ such that the isoperimetric profile of $(M, g)$ satisfies

$$
h(M, g ; s) \geq h\left(S^{n}(R), \text { can; } s\right), \quad \text { for all } s \in[0,1],
$$

where can is the canonical metric on the sphere $S^{n}(R)$ of radius $R$. Then, for $1 \leq p \leq n q /(n-q)$ and $p<\infty$,

$$
\forall f \in W^{1, q}(M), \quad\|f\|_{p} \leq S_{p, q}(M, g)\|d f\|_{q}+V(g)^{(1 / p-1 / q)}\|f\|_{q},
$$

where $S_{p, q}(M, g)=\left(V(g) / \operatorname{vol} S^{n}(1)\right)^{1 / p-1 / q} R \Sigma(n ; p, q)$.

PrOOF. One can approximate functions in $W^{1, q}$ by Morse functions. For a nonnegative function $f$ on $M$, and a point $A$ in $S^{n}(R)$, we define a function $f_{A}^{*}$ as follows. Let $B_{A}(t)$ be the geodesic ball centered at $A$ in $S^{n}(R)$, such that

$$
\operatorname{vol} B_{A}(t) / \operatorname{vol} S^{n}(R)=\operatorname{vol}\{f>t\} / \operatorname{vol} M \text {. }
$$

Define $f_{A}^{*}$ as

$$
f_{A}^{*}(x)= \begin{cases}t & \text { if } x \in \partial B_{A}(t), 0 \leq t \leq \sup f \\ 0 & \text { if } x \notin B_{A}(0)\end{cases}
$$


Now, let $f$ be a Morse function with $\int_{M} f v_{g}=0$. We write $f=f_{+}-f_{-}$, where $f_{+}$(resp. $f_{-}$) is the positive (resp. negative) part of $f$. We define the function $f^{*}$ by

$$
f^{*}=\left(f_{+}\right)_{N}^{*}-\left(f_{-}\right)_{S}^{*}
$$

where $N$ (resp. $S$ ) is the north (resp. south) pole of $S^{n}(R)$.

Equality (4) implies that the functions $f$ and $f^{*}$ are equi-measurable (up to normalizing constants). In particular,

$$
\left\{\begin{array}{l}
\text { (i ) } \int_{M^{*}} f^{*}=\int_{M} f=0, \quad M^{*}=S^{n}(R), \\
\text { (ii) } \operatorname{vol}(M)^{-1} \int_{M}|f|^{p}=\operatorname{vol}\left(M^{*}\right)^{-1} \int_{M^{*}}\left|f^{*}\right|^{p}
\end{array}\right.
$$

(see [Ba] or apply the co-area formula [Bé-Me, Appendix A]).

Let $h\left(\right.$ resp. $\left.h^{*}\right)$ denote either $f_{+}$or $f_{-}$(resp. $f_{+}^{*}$ or $f_{-}^{*}$ ). Since $f$ is a Morse function, we can easily apply the co-area formula to $h$. This gives

$$
\int_{\{h>t\}}|d h|^{q} v_{g}=\int_{t}^{\infty}\left(\int_{h^{-1}(t)}|d h|^{q-1} d \sigma\right) d t
$$

where $d \sigma$ is the Riemannian measure on $h^{-1}(t)$.

Hölder's inequality gives

(b) $\quad \operatorname{vol} h^{-1}(t) \leq\left(\int_{h^{-1}(t)}|d h|^{q-1} d \sigma\right)^{1 / q} \cdot\left(\int_{h^{-1}(t)}|d h|^{-1} d \sigma\right)^{(q-1) / q}$

Let $V(t)=\operatorname{vol}\{h>t\}$. By the co-area formula, we also have

$$
V^{\prime}(t)=-\int_{h^{-1}(t)}|d h|^{-1} d \sigma .
$$

The assumption on the isoperimetric function of $(M, g),(4)$ and the definition of $h^{*}$, imply that

$$
\operatorname{vol} h^{-1}(t) / \operatorname{vol} M \geq \operatorname{vol} h^{*-1}(t) / \operatorname{vol} S^{n}(R)
$$

(we also use the fact that the geodesic balls minimize the volume of the boundary, among domains with given volume on $S^{n}(R)$ ).

Relations (b)-(d) and (4) yield

$$
\int_{h^{-1}(t)}|d h|^{q-1} d \sigma / \operatorname{vol} M \geq \int_{h^{*-1}(t)}\left|d h^{*}\right|^{q-1} d \sigma / \operatorname{vol} S^{n}(R)
$$

( $h^{*}$ being radial, equality holds in (b) applied to $h^{*}$ ). From equality (a), we finally conclude that

$$
\|d h\|_{q}^{q} / \operatorname{vol} M \geq\left\|d h^{*}\right\|_{q}^{q} / \operatorname{vol} S^{n}(R),
$$

i.e. "symmetrization decreases energy" (up to normalizing constants).

From (5) and (6), we conclude that the number

$$
S_{p, q}(M, g)=\sup \left\{\|f\|_{p} /\|d f\|_{q}: f \in W^{1, q}, f \neq 0, \int_{M} f=0\right\}
$$

satisfies the inequality

$$
S_{p, q}(M, g) \leq\left(\operatorname{vol}(M) / \operatorname{vol}\left(S^{n}(R)\right)\right)^{1 / p-1 / q} S_{p, q}\left(S^{n}(R), \text { can }\right) .
$$


Recalling that $|d f|$ is the norm of the one-form $d f$ in the dual metric, an easy homogeneity argument gives

$$
S_{p, q}(M, g) \leq\left(\operatorname{vol}(M, g) / \operatorname{vol} S^{n}(1)\right)^{1 / p-1 / q} R \Sigma(n ; p, q) .
$$

In order to finish the proof of Theorem 3, it suffices to take $f \in W^{1, q}$ and to apply (7) to $f_{1}=f-V(g)^{-1} \int_{M} f v_{g}$,

$$
\left\|f_{1}\right\|_{p} \leq S_{p, q}\left\|d f_{1}\right\|_{q}=S_{p, q}\|d f\|_{q}
$$

and to notice that

$$
\|f\|_{p} \leq\left\|f_{1}\right\|_{p}+V(g)^{(1 / p-1 / q)}\|f\|_{q}
$$

by Hölder's inequality.

The interesting case for our purposes is when $q=2$ and $p=2 n /(n-2)$ (if $n \geq 3$ ) or $p \in[1, \infty[$ (if $n=2$ ). However, the case $p=q=2$ also deserves special attention, as the following corollary shows.

8. COROllary. Let $(M, g)$ be an $n$-dimensional closed $C^{\infty}$ Riemannian manifold such that $h(M, g ; s) \geq h\left(S^{n}(R)\right.$, can; $\left.s\right)$. Let $\lambda_{2}(M, g)$ be the first nonzero eigenvalue of the Laplacian on $(M, g)$. Then

$$
\lambda_{2}(M, g) \geq \lambda_{2}\left(S^{n}(R), \text { can }\right)=n R^{-2} .
$$

PROOF. Apply inequality (7) (proof of Theorem 3), with $p=q=2$ : for any $f \in W^{1,2}, \int_{M} f=0$, we have

$$
\|f\|_{2} \leq R \Sigma(n ; 2,2)\|d f\|_{2} .
$$

By the variational characterization of $\lambda_{2}$, we have $\lambda_{2}\|f\|_{2}^{2} \leq\|d f\|_{2}^{2}$ for any such $f$; similarly, we find that $\Sigma(n ; 2,2)=n^{-1 / 2}$. The corollary follows.

9. Comments. As was pointed out by E. Bombieri, H. Federer and W. Fleming, the Sobolev constant of the embedding $W^{1,1} \rightarrow L^{n /(n-1)}$ can be estimated in terms of the isoperimetric constant $I(M, g)$ which we defined in Appendix I. More precisely, if $C(M, g)$ is the least constant such that

$$
\forall f \in W^{1,1}, \quad \inf _{a \in \mathbf{R}}\|f-a\|_{n /(n-1)} \leq C(M, g)\|d f\|_{1},
$$

we have the inequality [Ch, Chapter IV],

$$
1 / 2 I(M, g) \leq C(M, g) \leq 1 / I(M, g) .
$$

In [Li], P. Li used inequality (10) to estimate the Sobolev constants of the embedding $W^{1,2} \rightarrow L^{p}(p=2 n /(n-2)$ if $n \geq 3 ; p \in[1, \infty[$ when $n=2)$.

In [Ga3], S. Gallot used $I(M, g)$ and J. Cheeger's isoperimetric constant $h_{C}(M, g)$ (see Appendix I) for the same purposes. His method however is different from that of $\mathrm{P}$. $\mathrm{Li}$; it does not rely on (10), but on symmetrization. One can improve S. Gallot's method (still using $I(M, g)$ ) by using a symmetrization associated with a "double-disk" as in [Bé-Ga].

In order to obtain explicit bounds for the Sobolev constants, one still has to estimate $I(M, g)$ and $h_{C}(M, g)$ from below, in terms of the geometry of $(M, g)$. In $[\mathbf{L i}], \mathrm{P}$. Li used estimates of $I(M, g)$ given by Ch. Croke $[\mathbf{C r}$ or Ch, Chapter V]. In [Ga2, 3], S. Gallot applied the isoperimetric estimates given in $[\mathbf{G a 1} \mathbf{1}$. 
It follows from Appendix I that given an isoperimetric estimator for $(M, g)$, one has lower bounds for $I(M, g)$ and $h_{C}(M, g)$, and conversely. From this point of view, Theorem 3 is sharper and more conceptual than [Li and Ga3]. Another advantage of Theorem 3 is that it gives bounds for the Sobolev constant of the embedding $W^{1, q} \rightarrow L^{p}$, for all $p$ such that $1 \leq p \leq n q /(n-q)$ and $p<\infty$; these bounds are sharper than the ones obtained by applying Hölder's inequality to the embedding $W^{1, q} \rightarrow L^{n q /(n-q)}(q<n)$, as Corollary 8 shows.

The proof of Theorem 3 follows classical ideas. We gave it with full details for the sake of completeness. The idea of using symmetrization for estimating the Sobolev constants was introduced by Th. Aubin and G. Talenti independently, for the embedding $W^{1, q}\left(\mathbf{R}^{n}\right) \rightarrow L^{p}\left(\mathbf{R}^{n}\right)$ (see [Au1, Ta, and Bé-Me] for a particular case of Theorem 3 ).

Using [Bé, Chapter IV], one can generalize Theorem 3 to other isoperimetric estimators. Estimating the Sobolev constants then reduces to a 1dimensional problem (compare with [Au1, Chapter 2]).

Appendix VII: Symmetrization of elliptic or parabolic inequalities. In this appendix, we show how one can estimate $\|f\|_{\infty}^{2} V(g) /\|f\|_{2}^{2}$, for a nonnegative solution $f$ of the inequality $\Delta f \leq a f$, on a closed Riemannian manifold $(M, g)$. We also state a theorem concerning the heat kernel of $(M, g)$.

We assume throughout that the isoperimetric profile $h(M, g ; s)$ (see Appendix I) satisifes the inequality

$$
\text { for any } s \in[0,1], \quad h(M, g ; s) \geq h\left(S^{n}(R), g ; s\right),
$$

where $R$ is some positive number (see Appendix I, Theorem 2).

In the context of Appendix II, $f$ is the norm $|s|$ of a $C^{\infty}$ section of a Riemannian fiber bundle $E$ over $M$. For $\varepsilon>0$, define $|s|_{\varepsilon}(x)=\left(|s(x)|^{2}+\right.$ $\left.\varepsilon^{2}\right)^{1 / 2}$. We then have $\Delta|s|_{\varepsilon} \leq a|s|_{\varepsilon}$. Since $|s|_{\varepsilon}$ is a $C^{\infty}$ function, we can approximate it by Morse functions $u_{\varepsilon, p}$ such that $\left.\left|u_{\varepsilon, p}-\right| s\right|_{\varepsilon} \mid \leq \varepsilon / p$ and $\left|\Delta\left(u_{\varepsilon, p}-|s|_{\varepsilon}\right)\right| \leq \varepsilon / p$. We conclude that

$$
\Delta u_{\varepsilon, p} \leq \Delta|s|_{\varepsilon}+\frac{\varepsilon}{p} \leq\left(a+\frac{1}{p}\right)|s|_{\varepsilon} \leq \frac{p}{p-1}\left(a+\frac{1}{p}\right) u_{\varepsilon, p} .
$$

This implies that we can limit ourselves to $f$ a positive Morse function, at least for our purposes.

2. THEOREM. Let $(M, g)$ be a closed Riemannian manifold whose isoperimetric function satisfies (1). Let $f$ be a positive Morse function on $M$ which satisfies the inequality $\Delta f \leq a f$ for some number $a \geq 0$. Let $b=a R^{2}$, and let $\varphi_{b}$ denote the regular solution of the Cauchy problem

$$
\left\{\begin{array}{l}
y^{\prime \prime}(r)+(n-1) \operatorname{cotg} r y^{\prime}(r)+b y(r)=0, \quad 0 \leq r<\pi, \\
y(0)=1 .
\end{array}\right.
$$

Let $r_{b}$ denote the first positive zero of $\varphi_{b}$. Then

$$
\|f\|_{\infty}^{2} V(g) /\|f\|_{2}^{2} \leq \operatorname{vol}\left(S^{n}\right)\left[\operatorname{vol}\left(S^{n-1}\right) \int_{0}^{r_{b}} \varphi_{b}^{2}(r) \sin ^{n-1} r d r\right]^{-1}
$$

(recall that $V(g)=\operatorname{vol}(M, g))$. 
REMARK. Theorem 2 together with Theorem 2 of Appendix I gives an effective method for estimating the quantity $\|f\|_{\infty}^{2} V(g) /\|f\|_{2}^{2}$, by elementary numerical analysis.

PROOF OF THEOREM 2. We introduce the following notations.

- $F(t)=\operatorname{vol}\{x \in M: f(x)>t\}, F$ is a decreasing continuous function;

- $\bar{F}(\tau)=$ the inverse function of $F, \bar{F}$ satisfies $\operatorname{vol}\{x \in M: f(x)>\bar{F}(\tau)\}=\tau$;

- For $s \in[0,1]$, we define

$$
M(s)=\{x \in M: f(x)>\bar{F}(s V(g))\},
$$

the level set of $f$ whose relative volume is $s$,

$$
\Gamma(s)=\partial M(s), \quad U(s)=\int_{M(s)} f v_{g} .
$$

Recall the co-area formula [Bé-Me, Appendix A]

$$
\int_{M} h v_{g}=\int_{0}^{\infty}\left(\int_{f^{-1}(t)} h|d f|^{-1} d \sigma\right) d t
$$

where $\sigma$ is the Riemannian measure associated with the metric $g \mid f^{-1}(t)$ on $f^{-1}(t)$.

Applying (3) to $1_{\{f>t\}}$ and differentiating, we have

$$
F^{\prime}(t)=-\int_{f^{-1}(t)}|d f|^{-1} d \sigma .
$$

Applying (3) to $h=f^{p} 1_{M(s)}$ and using (a), we obtain

$$
\int_{M(s)} f^{p} v_{g}=\int_{0}^{s V(g)} \bar{F}(t)^{p} d t .
$$

Since $\Delta f \leq a f$, integrating over $M(s)$, applying Green's formula and using (a) gives

$$
a U(s) \geq \int_{M(s)} \Delta f v_{g}=\int_{\Gamma(s)}|d f| d \sigma \geq-(\operatorname{vol} \Gamma(s))^{2} / F^{\prime}(\bar{F}(s V(g))) .
$$

Since vol $M(s) / \operatorname{vol} M=s$, we can apply the isoperimetric inequality (1) (with $H(s)=h\left(S^{n}(R)\right.$, can; $\left.s\right)$ ); this gives

$$
a U(s) \geq-V(g)^{2} H^{2}(s) / F^{\prime}(\bar{F}(s V(g)))=-V(g)^{2} H^{2}(s) \bar{F}^{\prime}(s V(g)),
$$

where the second equality follows from the fact that $\bar{F}$ is the inverse function of $F$. Taking (b) with $p=1$ into account, we conclude that $U(s)$ satisfies

$$
\left\{\begin{array}{l}
H^{2}(s) \ddot{U}(s)+a U(s) \geq 0 \\
U(0)=0 \\
\dot{U}(0)=V(g)\|f\|_{\infty} .
\end{array}\right.
$$


Define the function $a(r)=(\sin (r / R))^{n-1}, 0 \leq r \leq R \pi$, and call $N$ (resp. $S$ ) the north (resp. south) pole of $S^{n}(R): N$ corresponds to $r=0$ and $S$ to $r=R \pi$. Let $\varphi$ be the regular solution of the Cauchy problem

$$
\left\{\begin{array}{l}
y^{\prime \prime}(r)+\left(a^{\prime}(r) / a(r)\right) y^{\prime}(r)+a y(r)=0 \quad \text { on }[0, R \pi[ \\
y(0)=\gamma>0
\end{array}\right.
$$

(regularity implies that $\varphi^{\prime}(0)=0$ ). As is easily seen, $\varphi$ decreases from $\gamma$ to 0 on $\left[0, r_{0}\right] \subset\left[0, R \pi\left[\right.\right.$, where $r_{0}$ is the first zero of $\varphi$ on $\mathbf{R}_{+}$. Call $f^{\wedge}$ the function defined on $S^{n}(R)$ by

$$
f^{\sim}(x)= \begin{cases}\varphi(\operatorname{dist}(N, x)), & \text { when } x \in B\left(N, r_{0}\right) \subset S^{n}(R) ; \\ 0, & \text { when } x \notin B\left(N, r_{0}\right) .\end{cases}
$$

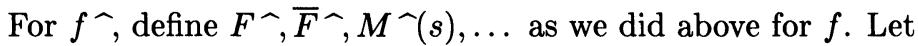

$$
s_{0}=\operatorname{vol} B\left(N, r_{0}\right) / \operatorname{vol} S^{n}(R) \text {. }
$$

Since $f^{-}$is a radial function, the preceding calculations give

$$
\left\{\begin{array}{l}
H^{2}(s) \ddot{U^{\curlyvee}}(s)+a U^{\curlyvee}(s)=0 \text { for } s \in\left[0, s_{0}[\right. \\
U^{\curlyvee}(0)=0 \\
\dot{U}^{\curlyvee}(0)=\operatorname{vol}\left(M^{\curlyvee}\right) \gamma=\operatorname{vol}\left(S^{n}(R)\right)\left\|f^{\wedge}\right\|_{\infty} .
\end{array}\right.
$$

The classical Sturm comparison trick shows that $U^{-}(s) / U(s)$ is nonincreasing in $\left[0, s_{0}\left[\right.\right.$; the function $U(s)$ is nondecreasing in $[0,1]$ and $U^{-}(s)$ is constant in $\left[s_{0}, 1\right]$. This proves

6. LeMMA. For all $s \in[0,1]$,

$$
V^{\curlyvee}(s)=\left(\operatorname{vol}\left(S^{n}(R)\right)\left\|f^{\wedge}\right\|_{\infty}\right)^{-1} U^{\curlyvee}(s) \leq\left(\operatorname{vol}(M)\|f\|_{\infty}\right)^{-1} U(s)=V(s) \text {. }
$$

It follows from equality (3) that

$$
U(s)=\int_{0}^{s \operatorname{vol}(M)} \bar{F}(t) d t \quad \text { and } \quad U^{\mathcal{}}(s)=\int_{0}^{s \operatorname{vol}\left(M^{\wedge}\right)} \bar{F}^{\curlyvee}(t) d t
$$

or equivalently,

$$
V(s)=\int_{0}^{s}\|f\|_{\infty}^{-1} \bar{F}(t \operatorname{vol} M) d t=\int_{0}^{s} y(t) d t
$$

and

$$
V^{\curlyvee}(s)=\int_{0}^{s}\left\|f^{\wedge}\right\|_{\infty}^{-1} \bar{F} \curlyvee\left(t \operatorname{vol} M^{\curlyvee}\right) d t=\int_{0}^{s} y \curlyvee(t) d t
$$

Lemma 6 says that the positive nonincreasing functions $y$ and $y$-satisfy

$$
\int_{0}^{s} y \widehat{\gamma}(t) d t \leq \int_{0}^{s} y(t) d t \quad \text { for all } s \in[0,1] .
$$

We can write

$$
\begin{aligned}
\int_{0}^{s}\left(y^{2}(t)-y^{-2}(t)\right) d t & =\int_{0}^{s}\left(y-y^{\curlyvee}\right)\left(y+y^{\curlyvee}\right)(t) d t \\
& =(y(0)+y\urcorner(0)) \int_{0}^{s_{1}}\left(y-y^{\curlyvee}\right)(t) d t
\end{aligned}
$$


for some $s_{1} \in[0, s]$, by the second mean value theorem. We conclude that $\int_{0}^{s} y^{-2}(t) d t \leq \int_{0}^{s} y^{2}(t) d t$ for all $s \in[0,1]$.

Using equality $(3 \mathrm{~b})$ again, with $p=2$, we finally obtain

$$
\left(\operatorname{vol}\left(S^{n}(R)\right)\left\|f^{\wedge}\right\|_{\infty}^{2}\right)^{-1}\left\|f^{\wedge}\right\|_{2}^{2} \leq\left(\operatorname{vol}(M)\|f\|_{\infty}^{2}\right)^{-1}\|f\|_{2}^{2} .
$$

To finish the proof of Theorem 2, take $\varphi(t)=\varphi_{b}(t / R)$ for $0 \leq t<R \pi$, note that $a^{\prime}(t) / a(t)=(n-1) \operatorname{cotg}(t / R) / R$ and use polar coordinates about the north pole $N$ to compute $\left\|f^{\wedge}\right\|_{2}$.

REMARK. Theorem 2 can be generalized to other isoperimetric estimators. The function $f$ is always compared to a radial function (see [Gam1] where Theorem 2 was proved for a "double disk" instead of a sphere as comparison model, compare with [Bé-Ga] where the "double disk" was first introduced, and to [Bé, Chapter IV; Gam2] where the sphere appears as comparison model).

Theorem 2 can be extended to deal with parabolic equations. For the proof of the following theorem we refer to [Bé-Ga, Bé-Be-Ga1 and Bé, Chapter V].

7. THEOREM. Let $(M, g)$ be an $n$-dimensional $C^{\infty}$ Riemannian manifold, which satisfies the isoperimetric inequality (1). Let $k(M, g ; t, x, y)$ denote the kernel of the heat operator $\exp (-t \Delta)$ on $C^{\infty}(M)$. Then

$$
\begin{aligned}
\int_{M} k(M, g ; t, x, x) v_{g} & \leq V(g) \sup _{x} k(M, g ; t, x, x) \\
& \leq \int_{S^{n}} k\left(S^{n}, \operatorname{can} ; t R^{-2}, x, x\right) d x
\end{aligned}
$$

where $\left(S^{n}\right.$, can) is the canonical unit sphere in $\mathbf{R}^{n+1}$.

REMARKS. (i) Let $m(k)$ denote the multiplicity of the eigenvalue $\lambda(k)=$ $k(k+n-1)$ of the Laplacian on $\left(S^{n}(1)\right.$, can $)$. The right-hand side of the inequalities in Theorem 7 is just

$$
\sum_{k=0}^{\infty} m(k) \exp \left(-t \lambda(k) / R^{2}\right) .
$$

This simple formula, together with Theorem 2 in Appendix I give a very effective method for estimating $k(M, g ; t, x, y)$.

(ii) An immediate consequence of Theorem 7 is that the isoperimetric inequality (1) implies lower bounds on the eigenvalues of $\Delta$, and upper bounds on the $L^{\infty}$-norms of the eigenfunctions of $\Delta$; see [Bé, Chapter V] and compare with [Ch, Chapter IV, VI, XII; Bé-Ber, Chapter 11].

\section{REFERENCES}

[Ad-Su] T. Adachi and T. Sunada, Energy spectrum of certain harmonic mappings, Compositio Math. 56 (1985), 153-170.

[Au1] Th. Aubin, Non linear analysis on manifolds, Monge-Ampère Equations, SpringerVerlag, 1982.

[Au2] _ Equations différentieles non linéaires et problème de Yamabe concernant la courbure scalaire, J. Math. Pures Appl. 55 (1976), 269-296. 
[Ba] C. Bandle, Isoperimetric inequalities and applications, Pitman Press, 1980.

[Ba-Pa] Ch. Bavard and P. Pansu, Sur le volume minimal de $\mathbf{R}^{2}$, Ann. Sci. École Norm. Sup. 19 (1986), 479-490.

[Bé] P. Bérard, Lectures on spectral geometry, $15^{\circ}$ Colóquio Brasileiro de Matemática, IMPA 1985 and Lecture Notes in Math., vol. 1207, Springer-Verlag, Berlin and New York, 1986.

[Bé-Be] P. Bérard and G. Besson, Integral bounds for some geometric invariants via the Bochner technique, Prépublication de l'Institut Fourier $n^{\circ} 78$, Grenoble, 1987.

[Bé-Ber] P. Bérard and M. Berger, Le spectre d'une variété riemannienne en 1982, Spectra of Riemannian Manifolds, Kaigai Publ., 1983, pp. 139-194, or [Bé, Appendix B].

[Bé-Be-Ga1] P. Bérard, G. Besson and S. Gallot, Sur une inégalité isopérimétrique qui généralise celle de P. Lévy-Gromov, Invent. Math. 80 (1985), 295-308.

[Bê-Ga] P. Bérard and S. Gallot, Inégalités isopérimétriques pour l'équation de la chaleur et application à l'estimation de quelques invariants, Exp. XV, Séminaire Goulaouic-MeyerSchwartz 83-84, École Polytechnique, Palaiseau, 1984.

[Bé-Ga] — Remarques sur quelques estimées géométriques explicites, C. R. Acad. Sci. Sér. I Math 297 (1983), 185-188.

[Bé-Me] P. Bérard and D. Meyer, Inégalités isopérimétriques et applications, Ann. Sci. École Norm. Sup 15 (1982), 513-542.

[B-G-M] M. Berger, P. Gauduchon and E. Mazet, Le spectre d'une variété riemannienne, Lecture Notes in Math., vol. 194, Springer-Verlag, Berlin and New York, 1971.

[Bo] S. Bochner, Vector fields and Ricci curvature, Bull. Amer. Math. Soc. 52 (1946), 776-797.

[Bo-Ya] S. Bochner and K. Yano Curvature and Betti numbers, Ann. of Math. Studies, no. 32, Princeton Univ. Press, 1953.

[Bou] J.-P. Bourguignon, Formules de Weitzenböck en dimension 4, Exposé $n^{\circ}$ XVI, A. Besse, Géométrie riemannienne de dimension 4, Cédic-Nathan, 1981.

[Bu-Ka] P. Buser and M. Karcher, Gromov's almost flat manifolds, Astérisque, 81, Soc. Math. France, Paris, 1981.

[Ch] I. Chavel, Eigenvalues in Riemannian geometry, Academic Press, New York, 1984.

[Che-Eb] J. Cheeger and D. Ebin, Comparison theorems in Riemannian geometry, American Elsevier, 1975.

[Co] G. Courtois, in preparation, 1986.

[Cr] Ch. Croke, Some isoperimetric inequalities and eigenvalue estimates, Ann. Sci. École Norm. Sup. Paris 13 (1981), 419-436.

[De] J.-P. Demailly, Majoration asymptotique de la cohomologie d'un fibré linéaire hermitien, Prépublication de I'Institut Fourier $n^{\circ} 25$, Grenoble, 1985. Champs magnétiques et inégalités de Morse pour la $d^{\prime \prime}$-cohomologie, Prépublication de l'Institut Fourier $n^{\circ} 37$, Grenoble, 1985.

[Do] J. Dodziuk, Vanishing theorems for square integrable harmonic forms, Proc. Indian Acad. Sci. Math. Sci. 90 (1981), 21-27.

[Ee] J. Eells, Elliptic operators on manifolds, Proc. Summer Course Complex Analysis (I.C.T.P. Trieste 1975), vol. 1, IAEA, 1976, pp. 95-152.

[Ee-Le] J. Eells and L. Lemaire, Selected topics in harmonic maps, CBMS Regional Conf. Ser. in Math., no. 50, Amer. Math. Soc., Providence, R. I., 1983.

[El] A. El Soufi, Thèse de $3 e$ cycle, Université de Grenoble, 1982.

[Ga1] S. Gallot, Inégalités isopérimétriques, courbure de Ricci et invariants géométriques. I and II, C. R. Acad. Sci. Sér. I Math. 296 (1983), 333-336 and 365-368.

[Ga2] _, A Sobolev inequality and some geometric applications, Spectra of Riemannian Manifolds, Kaigai Publ., 1983, pp. 45-55.

[Ga3] _ Bornes universelles pour des invariants géométriques, Séminaire Théorie Spectrale et Géométrie (Chambéry-Grenoble 1982-1983), Publications de l'Institut Fourier, Grenoble.

[Ga-Me] S. Gallot and D. Meyer, Opérateur de courbure et laplacien des formes différentielles d'une variété riemannienne, J. Math. Pures Appl. 54 (1975), 259-284.

[Gam1] N. Gamara, Thése de 3e cycle, Université de Savoie, Chambéry, 1983. 
[Gam2] N. Abdelmoula-Gamara, Symétrisation d'inéquations elliptiques et applications géométriques, Math. Z. (to appear).

[Gi-Tr] D. Gilbarg and N. S. Trudinger Elliptic partial differential equations of second order, Grundlehren Math. Wiss. vol 224, Springer-Verlag, Berlin and New York, 1977. 195.

[Gr1] M. Gromov, Curvature and Betti numbers, Comment. Math. Helv 56 (1981), 179-

[Gr2] — , P. Levy's isoperimetric inequality, Preprint, Inst. Hautes Études Sci., 1980.

[Gr3] _ Structure métriques pour les variétés riemanniennes, rédigé par J. Lafontaine et P. Pansu, Cédic-Nathan, 1981.

[Gr-La] M. Gromov and B. H. Lawson Positive scalar curvature and the Dirac operator on complete Riemannian manifolds, Inst. Hautes Études Sci. Publ. Math. 58 (1983), 83-196.

[H-S-U] H. Hess, R. Schrader and D. A. Uhlenbrock, Kato's inequality and the spectral distribution of Laplacians on compact Riemannian manifolds, J. Differential Geom. 15 (1980), 27-38.

[Hi] N. Hitchin, Harmonic spinors, Adv. in Math. 14 (1974), 1-55.

[Hu] D. Hulin, Pinching and Betti numbers, Ann. Global Anal. Geom. 3 (1985), 85-93.

[Ka] J.-L. Kazdan, Positive energy in general relativity, Séminaire Bourbaki, Exp. 593, Astérisque $n^{\circ}$ 92-93, 1982.

[Ko-No] S. Kobayashi and K. Nomizu, Foundations of differential geometry, vol. II, Interscience, 1969.

[La] B.-H. Lawson, Theory of gauge fields in four-manifolds, CBMS Regional Conf. Ser. in Math., no. 58, Amer. Math. Soc., Providence, R. I., 1984.

[Li] P. Li, On the Sobolev constant and the p-spectrum of a compact Riemannian manifold, Ann. Sci. École Norm. Sup. 13 (1980), 451-469. 7-9.

[Lz] A. Lichnerowicz, Spineurs harmoniques, C. R. Acad. Sci. Sér. I Math. 257 (1963),

[Me1] D. Meyer, Sur les variétés riemanniennes à opérateurs de courbure positif, C. R. Acad. Sci. Sér. I Math. 272 (1971), 482-485.

[Me2] __, Une inégalité de géométrie hilbertienne et ses applications à la géométrie riemannienne, Preprint 1984, Univ. Paris 7 and C. R. Acad. Sci. Sér. I Math. 295 (1982), 467-469.

[Me3] _ , Work in progress, 1986.

[Mi-Mo] M. Micallef and J.-D. Moore, Minimal two-spheres and the topology of manifolds with positive curvature on totally isotropic two-planes, Preprint, Univ. of California Santa Barbara, 1986.

[Mo] J.-D. Moore, On stability of minimal spheres and a two-dimensional version of Synge's theorem, Arch. Math. 44 (1985), 278-281.

[Pa] P. Pansu, Dégénérescence des variétés riemanniennes, Séminaire Bourbaki, Exposé 618, Novembre, 1983.

[Re-Si] M. Reed and B. Simon, Methods of modern mathematical physics, Academic Press, New York, 1978.

[Ro] S. Rosenberg, Semigroup domination and vanishing theorems (Proceedings 1987 Cornell Conf. on Geometry of Random Motion) Contemp. Math., vol. 73, Amer. Math. Soc., Providence, R. I., 1988, pp. 287-302.

[Sa] T. Sakai, Comparison and finiteness theorems in Riemannian geometry, Geometry of geodesics and related topics, Adv. Stud. Pure Math. 3 (1984), 125-181.

[Sc-Yau] R. Schoen and S.-T. Yau, Complete three-dimensional manifolds with positive Ricci curvature and scalar curvature, Seminar on Differential Geometry, Ann. of Math. Studies, no. 102, Princeton Univ. Press, 1982, pp. 209-227.

[Sh-So] B. Shiffman and A.-J. Sommese, Vanishing theorems in complex manifolds, Progress in Math., vol 56, Birkhäuser, Boston, 1985.

[Si1] Y.-T. Siu, Some recent results in complex manifold theory related to vanishing theorems for the semi-positive case, Arbeitstagung Bonn (1984), Lecture Notes in Math., vol 1111,Springer-Verlag, Berlin and New York, 1985, pp. 169-192.

[Si2] __, Asymptotic Morse inequalities for analytic sheaf cohomology, Séminaire Bourbaki, Exposé $n^{\circ}$ 666, Juin, 1986. 
[Ta] G. Talenti, Best constants in Sobolev inequality, Ann. Math. Pura Appl. 110 (1976), 353-372.

[Ur] H. Urakawa, Stability of harmonic maps and eigenvalues of the laplacian, Trans. Amer. Math. Soc. 301 (1987), 557-590.

[Wu] H. Wu, The Bochner technique, Proceedings 1980 Beijing symposium on Differential Geometry and Differential Equations. (S. S. Chern and Wu Wen-Tsün, eds.), Science Press and Gordon-Breach 1982, and The Bochner technique in differential geometry, Math. Rep., 1987.

[Yau1] S.-T. Yau, Seminar on differential geometry (S. T. Yau, ed.), Ann. of Math. Studies, no. 102, Princeton Univ. Press, 1982.

[Yau2] _ Differential geometry and complex analysis: a volume dedicated to the memory of H. E. Rauch (I. Chavel and H. M. Farkas, eds.), Springer, 1985.

NOTE ADDED IN PROOFS. The following references should be added at the end of $\S \mathrm{H}$.

S. Gallot, Isoperimetric inequalities based on integral norms of Ricci curvature, Astérisque (to appear).

to appear).

$\mathrm{S}$. Gallot and D. Meyer, D'un résultat hilbertien à un principe de comparaison entre spectres. Applications, Ann. Sci. Ecole Norm. Sup. (to appear).

Department of MAthematics, Institut Fourier, B. P. 74, 38402 St. MARtin D'HÈRES CEDEX, FRANCE 